NBER WORKING PAPER SERIES

\title{
ESTIMATING PROBABILITIES OF SUCCESS OF VACCINE AND OTHER ANTI-INFECTIVE THERAPEUTIC DEVELOPMENT PROGRAMS
}

\author{
Andrew W. Lo \\ Kien Wei Siah \\ Chi Heem Wong \\ Working Paper 27176 \\ http://www.nber.org/papers/w27176
}

\author{
NATIONAL BUREAU OF ECONOMIC RESEARCH \\ 1050 Massachusetts Avenue \\ Cambridge, MA 02138 \\ May 2020
}

We thank Informa for providing us access to their data and expertise and are particularly grateful to Will Akie, Christine Blazynski, Gabrielle Gessner, Mark Gordon, Michael Hay, Ian Lloyd, and Ryan Sasaki. We also thank Christine Blazynski, John Tedrow, the editor, associate editor, and reviewers for helpful comments on this manuscript. Research support from the MIT Laboratory for Financial Engineering and funding support from The Rockefeller Foundation are gratefully acknowledged. The views and opinions expressed in this article are those of the authors only, and do not necessarily represent the views and opinions of any institution or agency, any of their affiliates or employees, any of the individuals acknowledged above, or the National Bureau of Economic Research. Funding support from the MIT Laboratory for Financial Engineering is gratefully acknowledged, but no direct funding was received for this study and no funding bodies had any role in study design, data collection and analysis, decision to publish, or preparation of this manuscript. The authors were personally salaried by their institutions during the period of writing (though no specific salary was set aside or given for the writing of this manuscript).

At least one co-author has disclosed a financial relationship of potential relevance for this research. Further information is available online at http://www.nber.org/papers/w27176.ack

NBER working papers are circulated for discussion and comment purposes. They have not been peer-reviewed or been subject to the review by the NBER Board of Directors that accompanies official NBER publications.

(C) 2020 by Andrew W. Lo, Kien Wei Siah, and Chi Heem Wong. All rights reserved. Short sections of text, not to exceed two paragraphs, may be quoted without explicit permission provided that full credit, including $\odot$ notice, is given to the source. 
Estimating Probabilities of Success of Vaccine and Other Anti-Infective Therapeutic Development Programs

Andrew W. Lo, Kien Wei Siah, and Chi Heem Wong

NBER Working Paper No. 27176

May 2020

JEL No. C01,C13,I1,I11,I13,I15,I18,L65

\begin{abstract}
A key driver in biopharmaceutical investment decisions is the probability of success of a drug development program. We estimate the probabilities of success (PoSs) of clinical trials for vaccines and other anti-infective therapeutics using 43,414 unique triplets of clinical trial, drug, and disease between January 1, 2000, and January 7, 2020, yielding 2,544 vaccine programs and 6,829 nonvaccine programs targeting infectious diseases. The overall estimated PoS for an industry-sponsored vaccine program is $39.6 \%$, and $16.3 \%$ for an industry-sponsored antiinfective therapeutic. Among industry-sponsored vaccines programs, only 12 out of 27 disease categories have seen at least one approval, with the most successful being against monkeypox $(100 \%)$, rotavirus $(78.7 \%)$, and Japanese encephalitis $(67.6 \%)$. The three infectious diseases with the highest PoSs for industry-sponsored nonvaccine therapeutics are smallpox (100\%), cytomegalovirus (CMV) infection (31.8\%), and onychomycosis (29.8\%). Non-industry-sponsored vaccine and nonvaccine development programs have lower overall PoSs: $6.8 \%$ and $8.2 \%$, respectively. Viruses involved in recent outbreaks-Middle East respiratory syndrome (MERS), severe acute respiratory syndrome (SARS), Ebola, and Zika-have had a combined total of only 45 nonvaccine development programs initiated over the past two decades, and no approved therapy to date. These estimates offer guidance both to biopharma investors as well as to policymakers seeking to identify areas most likely to be underserved by private sector engagement and in need of public sector support.
\end{abstract}

Andrew W. Lo

MIT Sloan School of Management

100 Main Street, E62-618

Cambridge, MA 02142

and NBER

alo-admin@mit.edu

Kien Wei Siah

MIT Laboratory for Financial Engineering

100 Main Street

Cambridge, MA 02142

kienwei@mit.edu

\author{
Chi Heem Wong \\ MIT Laboratory for Financial Engineering \\ 100 Main Street \\ Cambridge, MA 02142 \\ chiheem@mit.edu
}

Supplementary Material is available at https://projectalpha.mit.edu/ 


\section{Introduction}

In this article, we provide estimates of the historical probabilities of success (PoSs) of clinical trials for vaccines and other therapeutic drugs for infectious diseases to inform discussions on the planning and financing of the fight against one of humanity's oldest foes. This is of particular importance in light of the recent havoc wreaked by severe acute respiratory syndrome coronavirus 2 (SARS-CoV-2), the virus that causes coronavirus disease (COVID-19).

While the PoSs of therapeutic drugs for various disease groups like oncology are welldocumented (Abrantes-Metz et al., 2004; DiMasi et al., 2010; Hay et al., 2014; MIT Laboratory for Financial Engineering, 2020; Smietana et al., 2016; Thomas et al., 2016; Wong et al., 2019a, 2019b), relatively little has been published on treatments for infectious diseases and vaccine development despite their importance (Davis et al., 2011; Pronker et al., 2013). Prior studies have focused on narrower subsets relevant to their specific interests and have relied on much more limited data sets. For example, Young et al. (2020) employed 10 to 25 data points per estimated value from the Bill and Melinda Gates Foundation to estimate the PoS of vaccines for neglected diseases, and DiMasi et al. (2020) reported PoS estimates on a perdrug basis using 2,575 trials for diseases of interest to the Gates Foundation. In contrast, we employ a much larger and broader data set of 16,328 unique clinical trials to estimate the PoSs of vaccines and nonvaccine therapeutics targeting 29 different infectious diseases using all available drug-indication pairs - a methodology that has been argued to be more relevant for evaluating drug development R\&D risk and productivity (Wong et al., 2019b).

Vaccination is commonly recognized as one of the most cost-effective public health measures for combatting infectious diseases (André, 2002; Ehreth, 2003; Kieny \& Girard, 2005; Organisation for Economic Co-operation and Development [OECD], 2013; Pronker et al., 2013; Rémy et al., 2015). In developed countries, routine prophylactic vaccination and effective treatment options have led to the control or complete elimination of several deadly infectious diseases through individual and herd immunity, preventing millions of deaths and untold suffering each year. This prophylaxis dramatically reduces the burden on the health care system and society as a whole. In addition, the deaths, hospitalizations, and treatment costs avoided by these measures have led to significant economic savings (Ehreth, 2003; Rémy et al., 2015; U.S. Department of Health and Human Services, 2017).

As technology continues to advance, one expects that the human species will be better able to cope with these diseases. The fact remains, however, that we still do not have effective treatments or vaccines for many infectious diseases. While the discovery of antibiotics has reduced the mortality rate of bacterial infection, and the development of the smallpox vaccine has led to the eradication of the devastating disease (World Health Organization, 1980), other infectious diseases, such as acquired immunodeficiency syndrome (AIDS) and malaria, still take the lives of tens of millions every year. According to the World Health

13 May 2020

(C) 2020 by Lo, Siah, and Wong

All Rights Reserved

Page 1 of 25 
Organization, there are currently only 26 infectious diseases that are preventable by available vaccines (World Health Organization, 2020).

By developing better risk measures for therapeutic development, we hope to facilitate greater investment, identification of underserved areas that require public sector support, and more efficient business and financing models in this critical field.

\section{Methods}

We apply the method of Wong et al. (2019b) to estimate the PoSs of drug development programs using historical clinical trial data. This method was also applied in Wong et al. (2019a) to investigate the clinical success rates of oncology development programs. We briefly describe this method, with parts reproduced from the aforementioned articles for expositional convenience.

A drug development program, also known as a drug development path, is the clinical investigation of the use of a drug for a disease. It typically consists of a sequence of clinical trials, separated into three phases. Phase 1 trials test mainly the safety and tolerance of a drug while phase 2 trials test the efficacy of the drug for a given indication. Phase 3 trials attempt to confirm the drug's efficacy for larger populations and against alternatives. Some trials involve the combination of two phases into a single protocol, denoted 'Phase 1/2' (a combination of Phases 1 and 2) and 'Phase 1/3' (a combination of Phases 2 and 3).

We say that a drug development program has reached phase $i$ if it is observed, or can be inferred, that there is at least one trial in phase $i$. It is possible that a clinical trial can be repeated in multiple development paths. In Figure 1, we show an example in which a single phase 1 trial for a drug is involved in four different development paths, each targeting a different disease. It is not uncommon that the result of the phase 1 trial is used as supporting evidence for the safe use of a drug, allowing that drug to be used for different indications without additional phase 1 testing. For example, hydroxychloroquine - already approved for the treatment of malaria - is being tested for effectiveness against COVID-19 without another phase 1 clinical trial. There also exist clinical trials where different drug combinations are tested for the same indication in different arms. Because of these multiplicities, computing PoSs cannot be done simply by dividing the number of phase $i+1$ trials by the number of phase $i$ trials for the same drug-indication pair - we need to identify specific drug development paths. 


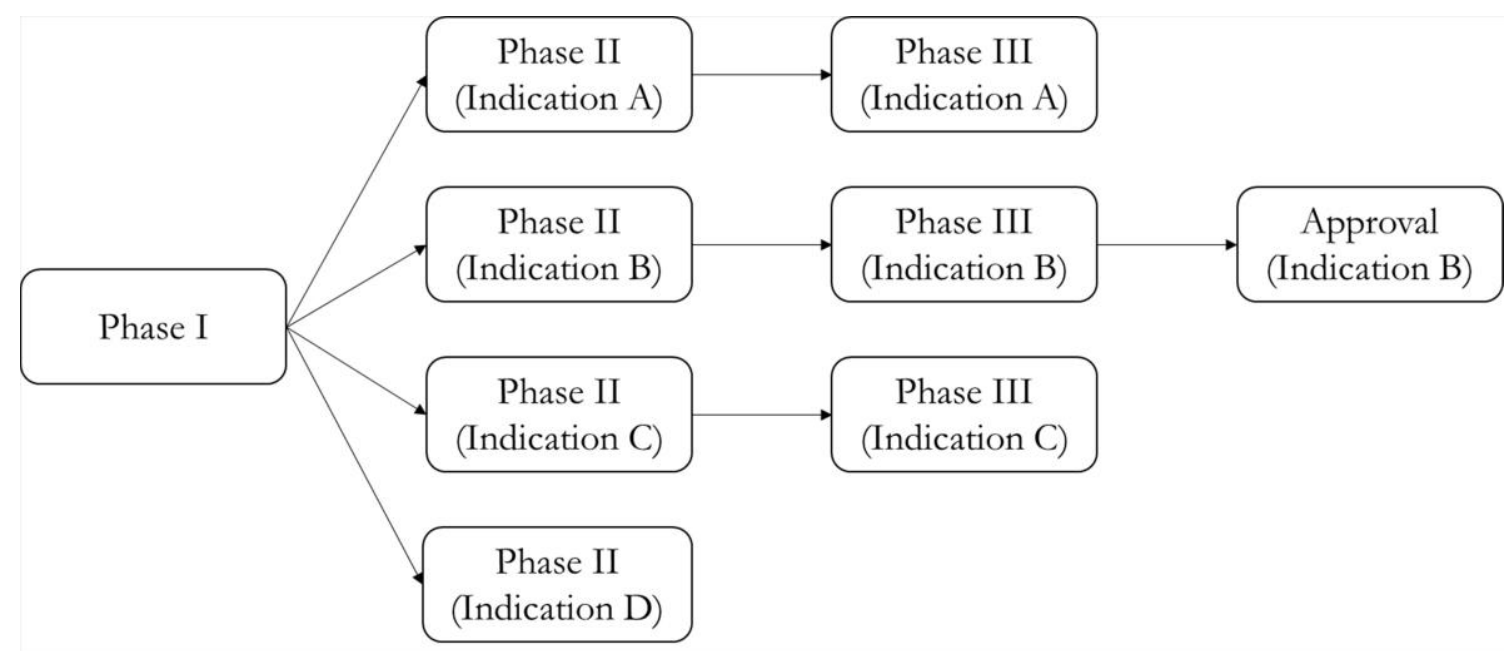

Figure 1. We define a drug development path as the development of a drug for a specific indication. A single clinical trial can belong to multiple drug development programs. We illustrate a hypothetical example where 4 drug development paths, all using the same drug, share the same phase 1 clinical trial.

Specifically, we make the assumption that each program must transition from phase 1 to phase 2 to phase 3 to approval in this order, and model the possible states in a drug development program as a Markov chain shown in Figure 2.

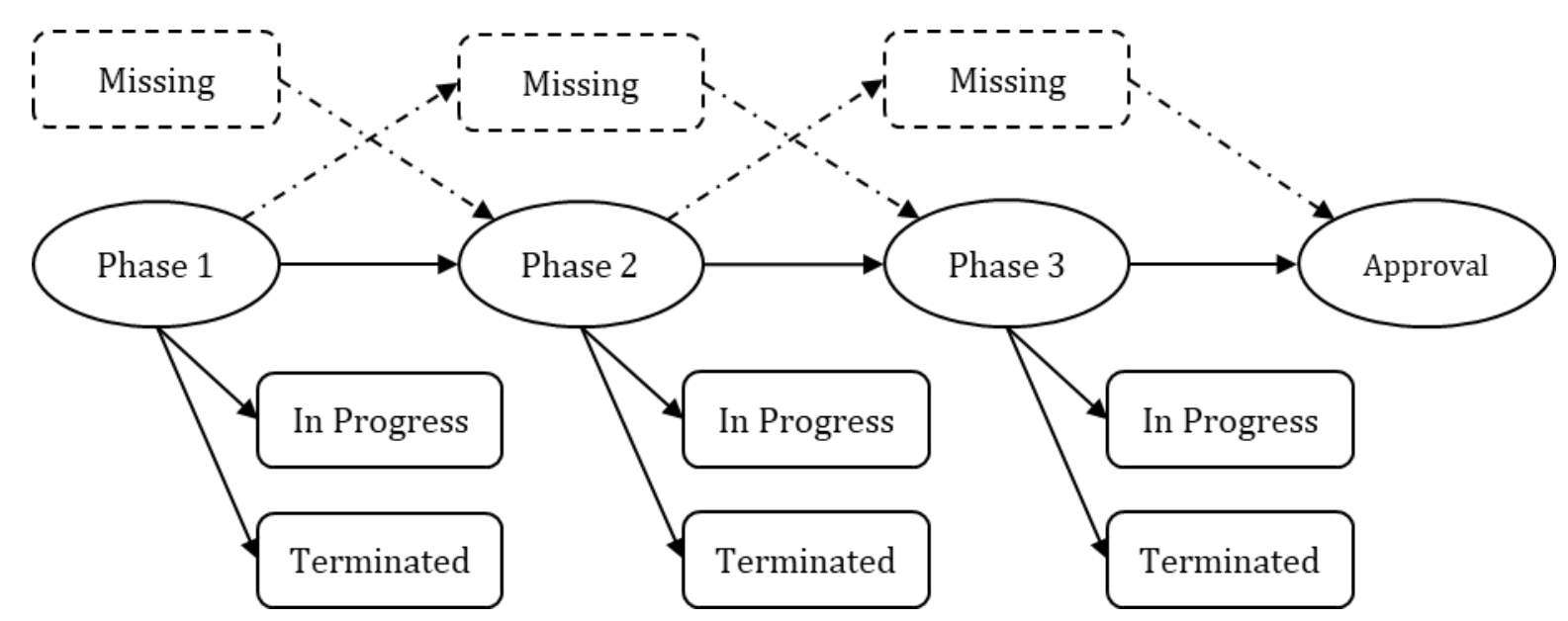

Figure 2. Observed and unobserved states in a drug development, from phase 1 to approval. A drug development is in phase $i$ if it has at least one trial in phase $i$. The 'missing' states represent phases where we do not observe any clinical trial in that phase for the drug-indication pair, but where we know must have occurred. Every drug development path in our study must start from phase 1 (or 'missing' phase 1) and end up in one of the nodes labeled as 'in progress', 'terminated' or 'approval'.

We infer missing transitions in the development paths arising from incomplete records. This is plausible since each of these stages involves distinct predefined tests, all of which are required by regulators in any new drug application (NDA). If we observe data for phases 1 and 3 but not phase 2 trials for a given drug-indication pair, our idealized process implies that there was at least one phase 2 trial that occurred, but is missing from our dataset. Accordingly, we impute the successful completion of phase 2 in these cases. There exist some 
rare cases where phase 2 trials are skipped, as with the example of aducanumab (BIIB037), Biogen's Alzheimer's candidate, as reported by Root (2014). Since skipping phase 2 trials is motivated by compelling phase 1 data and is approved by the regulatory authorities, imputing the successful completion of phase 2 trials in these cases to trace drug development paths is a reasonable approximation. We make the standard assumption that phase $1 / 2$ and phase $2 / 3$ trials are to be considered as phase 2 and phase 3 , respectively.

We call the estimated probability of a drug development program transitioning from phase $i$ to phase $i+1$ the "phase $i$ PoS", and the "estimated overall PoS" is defined as the estimated probability of a drug development program going from phase 1 to regulatory approval in at least one country. To simplify terminology, we will henceforth omit the qualifier "estimated" when referring to the PoS, so it should be understood that all PoS values reported in this article are statistical estimates of unobservable population parameters.

The probability of a drug development program transitioning from phase $i$ to phase $j$ $\left(\mathrm{PoS}_{i j}\right)$ can be computed using the simple ratio $\mathrm{N}_{j} / \mathrm{N}_{i}$, where $\mathrm{N}_{i}$ is the number of drug development programs that have reached phase $i$ (where $i=1,2,3$ ) of the drug development process and are not in active development between phase $i$ and phase $j$ (where $j=2,3$, or " $\mathrm{A}$ " which denotes regulatory approval, $i<j$ ), and $\mathrm{N}_{j}$ is the number of drug development programs among the former that made it to phase $j$. $\mathrm{PoS}_{1 \mathrm{~A}}$ is also known as the "overall PoS". We provide simple numerical examples in the Appendix (Section A1) to clarify our algorithms.

The estimated probability of a drug development program transitioning from phase 1 to approval - estimated directly using the method described above-is called the 'path-bypath' estimate of the overall PoS, and is reported for all PoS calculations. Our method of inferring unobserved states and computing the PoS using the simple ratio defined above applies to both vaccines and nonvaccine therapeutics. In fact, since it is common for vaccine candidates to skip phase 1 and move directly to phase 2 or 3 based on initial safety of the vaccine base (e.g., egg, etc.) after changing the virus within it, filling in unobserved phases will lead to a more accurate PoS.

It should be emphasized that because of the treatment of in-progress drug development programs, path-by-path PoS estimates are not multiplicative, i.e., $\operatorname{PoS}_{12} \times$ $\mathrm{PoS}_{23} \times \operatorname{PoS}_{3 \mathrm{~A}} \neq \mathrm{PoS}_{1 \mathrm{~A}}$. In contrast, the 'phase-by-phase' estimates used in prior studies (DiMasi et al., 2010, 2020; Hay et al., 2014; Thomas et al., 2016) do multiply, i.e., $\mathrm{PoS}_{12} \times \mathrm{PoS}_{23} \times \mathrm{PoS}_{3 \mathrm{~A}}=\mathrm{PoS}_{1 \mathrm{~A}}$. The latter two studies do not fill in unobserved clinical development phases. We elaborate on the differences between the path-by-path and phaseby-phase methods in the Appendix (see Section A2).

We compute the path-by-path POS using an algorithm that recursively considers all possible drug-indication pairs and determines the maximum observed phase. As the Markov chain model implies, reaching phase $i$ would imply that all prior phases were completed. To determine if a drug development program has been terminated in the last observed phase

13 May 2020

(C) 2020 by Lo, Siah, and Wong

Page 4 of 25

All Rights Reserved 
or is still ongoing, we use a simple heuristic: If the time elapsed between the end date of the most recent phase $i$ and the end of our sample exceeds a certain threshold $t_{i}$, we conclude that the trial has terminated. Based on practical considerations, we set $t_{i}$, to be 360,540 , and 900 days for phases 1,2 , and 3, respectively. For example, we assume that it takes approximately 6 months to prepare documents for an NDA filing after a phase 3 trial has been completed. Since the FDA has a 6-month period to decide if it wishes to follow up on a filing, and an additional 18 months to deliver a verdict, this places the overall time between phase 3 and Approval to about 30 months, hence we set $t_{3}=900$ days. Based on these criteria, we will consider only drug development programs that have seen at least one trial with a definite outcome in the PoS computations. More detailed exposition of and pseudo-code for this algorithm can be found in Wong et al. (2019b).

\section{Data}

We extracted clinical trials metadata from the January 7,2020, snapshots of Citeline's PharmaProjects and TrialTrove databases, provided by Informa Pharma Intelligence. These data are widely available commercially as well as through an academic license. Clinical trial metadata was retrieved from the TrialTrove database while the approval data was obtained from the PharmaProjects database, both of which are required to identify the drug development programs. In addition to incorporating multiple data streams, including nightly feeds from official sources such as ClinicalTrials.gov, Citeline contains data from primary sources such as institutional press releases, financial reports, study reports, and drug marketing label applications, and secondary sources such as analyst reports by consulting companies. Secondary sources are particularly important for reducing potential biases that may arise from the tendency of organizations to report only successful trials, especially those prior to the FDA Amendments Act of 2007 which requires all clinical trials to be registered and tracked via ClinicalTrials.gov. These databases contain information from both US and non-US sources. We consider a drug approved if it is approved in any country. All clinical trials used in this analysis have end dates after January 1, 2000, and start dates before January 7, 2020.

We filter our data to include only trials that have been tagged by Citeline as being in the 'Infectious Disease' or 'Vaccines (Infectious Diseases)' therapeutic areas. The vaccine types and diseases are provided by the databases. The database encodes each unique triplet of trial identification number, drug, and disease as a data point. Therefore, a single trial may appear as multiple data points. Since the two therapeutic areas may overlap in data points, we define clinical trials that are involved in any vaccine development as part of a 'vaccine' development program. In addition, we process the data such that more specific diseases (e.g., rabies) can be identified instead of broad vaccine classes (e.g., vector-borne disease vaccines). Clinical trials that are not involved in any vaccine development program will be deemed to be part of a 'nonvaccine' drug development program. We derive 43,414 data

13 May 2020

(C) 2020 by Lo, Siah, and Wong

All Rights Reserved

Page 5 of 25 
points in total. We define an 'industry-sponsored' development program as one where there is at least one commercial company involved in any stage of clinical development. The complement-in which there is no commercial company involved in any stage of the vaccine or drug development program-shall be referred to as 'non-industry-sponsored'. Given these definitions, a drug or vaccine development program (and the clinical trials in the program) can belong to only one of these mutually-exclusive sets: industry-sponsored vaccines, industry-sponsored nonvaccine therapeutics, non-industry-sponsored vaccines, and, non-industry-sponsored nonvaccine therapeutics.

The vaccines in TrialTrove are identified by broad categories such as "respiratory vaccines," "other viral vaccines," or "hepatitis vaccines." We attempt to infer the diseases targeted by the vaccines by cross-referencing the disease tags for each clinical trial. For example, a clinical trial may be tagged with both "hepatitis vaccines" and "HBV," allowing us to conclude that the vaccine is indicated for HBV (hepatitis B virus). Those clinical trials that have only vaccine tags will have their disease labeled as "others." Manual inspection of some of the clinical trial titles shows that this category includes diseases such as measles and tuberculosis.

We plot the number of development programs known to start in each month from January 2000 through December 2019 in Figure 3. There are 1,838 and 706 industrysponsored and non-industry-sponsored vaccine development programs, respectively, and, 3,851 and 2,978 industry-sponsored and non-industry-sponsored nonvaccine drug development programs targeting infectious diseases, respectively. As can be seen from Figure 3a, the number of industry-sponsored clinical programs attempting to treat infectious diseases is often greater than the number of vaccine development programs. We see a precipitous fall in the number of infectious disease treatment development programs initiated between late 2018 and mid-2019, which is likely to be related to declining investment in the research and development (R\&D) of novel antibiotics, precipitated by the closure of antibiotics biotechnology firms and the withdrawal of pharmaceutical companies from the antibiotics business (Hu, 2018; Langreth, 2019).

Between January 2000 and June 2011, the number of non-industry-sponsored vaccine development programs initiated is on par with the number of non-industrysponsored, nonvaccine anti-infective drug development programs initiated (see Figure 3b). However, the number of nonvaccine drug development programs initiated begin to outpace the number of vaccine development programs after January 2012, and experience a rapid boom between mid-2015 and mid-2018 before declining rapidly between October 2018 and January 2019.

13 May 2020

(C) 2020 by Lo, Siah, and Wong All Rights Reserved

Page 6 of 25 


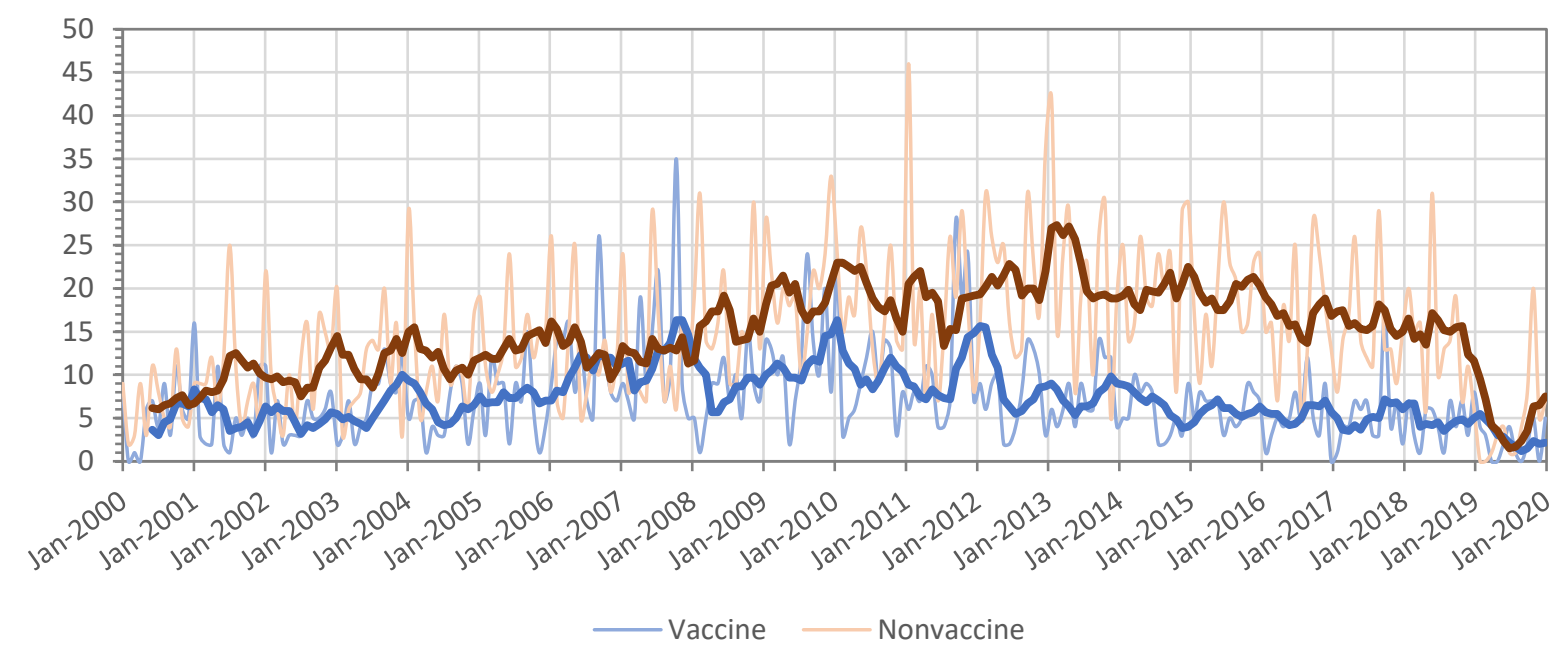

(a) Number of industry-sponsored development programs initiated

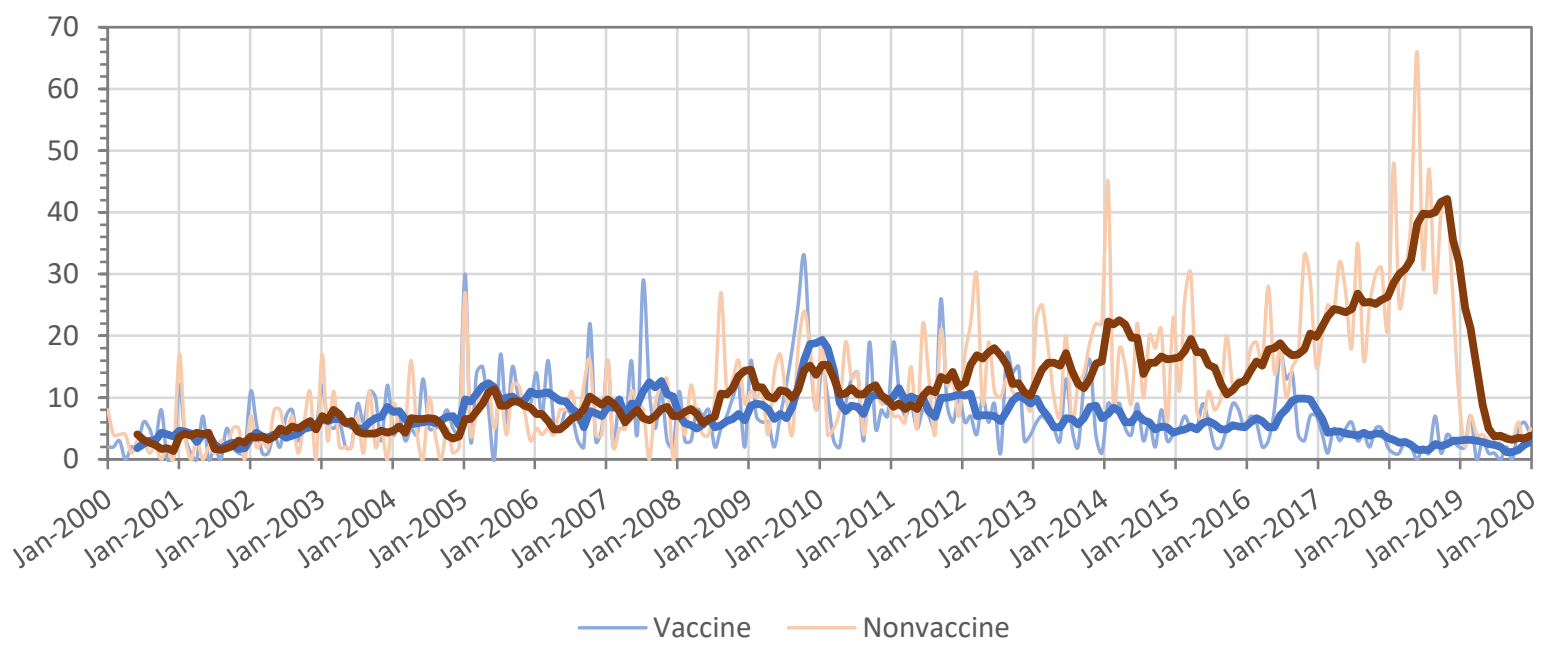

(b) Number of non-industry-sponsored development programs initiated

Figure 3. The number of development programs initiated per month from January 2000 through December 2019 in the areas of vaccine and nonvaccine treatment for infectious diseases (thin, light colored lines). The darker, thicker lines represent the 6-month moving average of the individual series.

\section{Results}

\subsection{Vaccines}

Overall, 2,544 vaccine development programs are observed in our data set, of which 1,838 are sponsored by industry and 706 do not involve any industry sponsor in any stage of development. For industry-sponsored drug development programs, respiratory infections is the most actively researched vaccine category, comprising $34.8 \%(n=640)$ of all vaccine development programs (see Figure 4). HBV and human immunodeficiency virus (HIV) vaccines represent $11.6 \%(n=213)$ and $9.8 \%(n=181)$ of all vaccine development programs, 
respectively, whereas intra-abdominal infections, monkeypox, and severe acute respiratory syndrome (SARS) vaccines are the least researched fields, with only one development path observed per disease.

A similar pattern can be seen for the non-industry-sponsored vaccine development programs; excluding the others category, the top three most researched vaccine categories are also respiratory infections (24.8\%), HIV (20.4\%), and HBV (8.2\%), whereas Middle East respiratory syndrome (MERS) and SARS are the least researched diseases with one development program each.

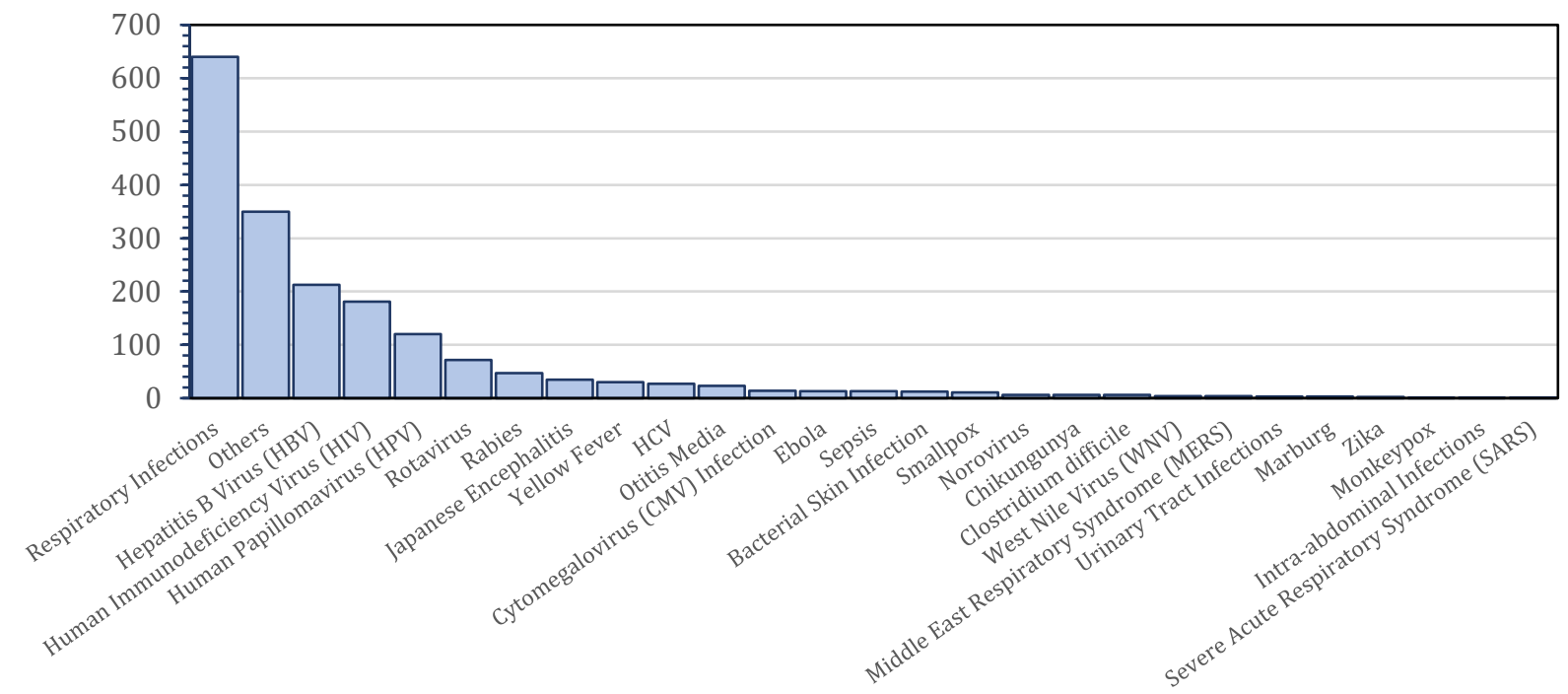

(a) Number of industry-sponsored vaccine development programs

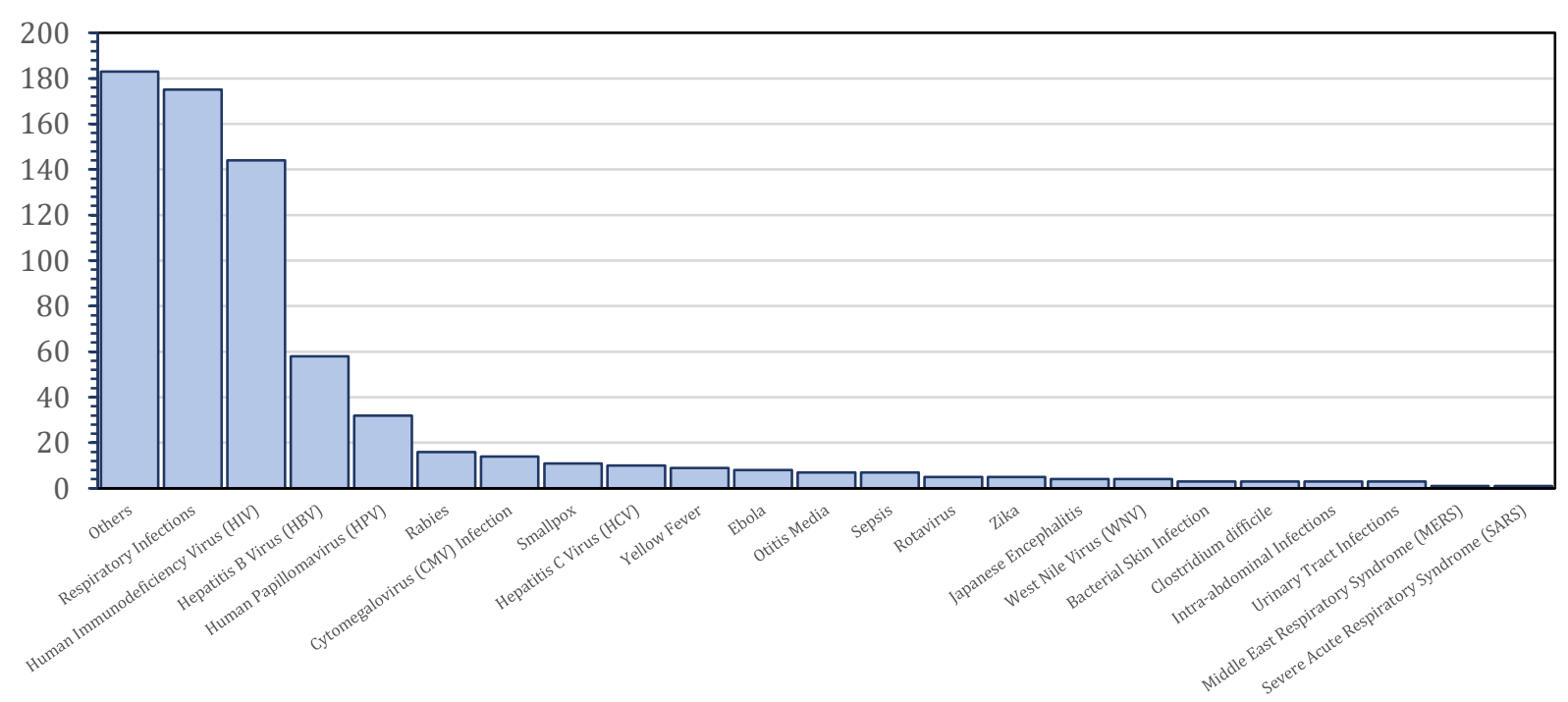

(b) Number of non-industry-sponsored vaccine development programs

Figure 4. Number of vaccine development programs observed for each vaccine type. HBV, hepatitis $B$ virus; HCV, hepatitis $C$ virus; HIV, human immunodeficiency virus; HPV, human papillomavirus. 
From Table 1, we can see that the overall PoS for industry-sponsored vaccine development programs is $39.6 \%$ (standard error, or $\mathrm{SE}=1.2 \%$ ), which is substantially higher than the average overall PoS of $11.0 \%$ (SE $=0.2 \%$ ) across all industry-sponsored drug development programs (see Table 10 in the Appendix). These findings are largely in line with the results of Wong et al. (2019b), who first observed this trend, and of DiMasi et al. (2020), despite the fact that the latter computed their estimates using a different method (a "phaseby-phase" approach) and considered only lead indications. We estimate $\mathrm{PoS}_{12}, \mathrm{PoS}_{23}$, and $\mathrm{PoS}_{3 \mathrm{~A}}$ to be $82.5 \%$ (SE $=0.9 \%$ ), 65.4\% (SE $=1.3 \%$ ), and 80.1\% (SE $=1.4 \%$ ), respectively.

Across all industry-sponsored vaccine development programs, we can see that monkeypox vaccines have had the most developmental success, followed by rotavirus and Japanese encephalitis vaccines (see Table 1). Their overall success rates are 100\% (SE = $0.0 \%$ ), 78.7\% (SE $=5.2 \%$ ), and 67.6\% (SE $=8.0 \%$ ), respectively. The overall PoS for monkeypox is based on only one sample. Only 12 diseases out of the 27 disease categories with at least one development path observed have seen at least one approved vaccine.

Table 1. The probabilities of success (PoSs) of industry-sponsored vaccine drug development programs. A: regulatory approval; P1: phase 1; P2: phase 2; P3: phase 3; SE = standard error.

\begin{tabular}{|c|c|c|c|c|c|c|c|c|c|c|c|c|c|c|}
\hline Disease & P1 Paths & $\mathbf{P o S}_{12}$ & SE & P2 Paths & $\mathrm{PoS}_{23}$ & SE & $\mathbf{P o S}_{2 \mathrm{~A}}$ & SE & P3 Paths & $\mathrm{PoS}_{3 \mathrm{~A}}$ & SE & Paths & $\operatorname{PoS}_{1 \mathrm{~A}}$ & SE \\
\hline Bacterial Skin Infection & 12 & 83.3 & 10.8 & 7 & 14.3 & 13.2 & 0.0 & 0.0 & 1 & 0.0 & 0.0 & 9 & 0.0 & 0.0 \\
\hline Chikungunya & 6 & 83.3 & 15.2 & 0 & - & - & - & - & 0 & - & - & 1 & 0.0 & 0.0 \\
\hline Clostridium difficile & 6 & 100.0 & 0.0 & 6 & 33.3 & 19.2 & 0.0 & 0.0 & 0 & - & - & 4 & 0.0 & 0.0 \\
\hline Cytomegalovirus Infection (CMV) & 14 & 57.1 & 13.2 & 3 & 33.3 & 27.2 & 0.0 & 0.0 & 0 & - & - & 8 & 0.0 & 0.0 \\
\hline Ebola & 13 & 53.8 & 13.8 & 7 & 57.1 & 18.7 & 28.6 & 20.2 & 2 & 100.0 & 0.0 & 11 & 18.2 & 11.6 \\
\hline Hepatitis B Virus (HBV) & 213 & 94.8 & 1.5 & 187 & 74.9 & 3.2 & 54.5 & 3.7 & 132 & 77.3 & 3.6 & 190 & 53.7 & 3.6 \\
\hline Hepatitis C Virus (HCV) & 27 & 70.4 & 8.8 & 15 & 0.0 & 0.0 & 0.0 & 0.0 & 0 & - & - & 23 & 0.0 & 0.0 \\
\hline Human Immunodeficiency Virus (HIV) & 181 & 65.2 & 3.5 & 95 & 36.8 & 4.9 & 0.0 & 0.0 & 21 & 0.0 & 0.0 & 144 & 0.0 & 0.0 \\
\hline Human Papillomavirus (HPV) & 120 & 88.3 & 2.9 & 69 & 52.2 & 6.0 & 36.2 & 6.1 & 30 & 83.3 & 6.8 & 77 & 32.5 & 5.3 \\
\hline Intra-abdominal Infections & 1 & 100.0 & 0.0 & 1 & 100.0 & 0.0 & 0.0 & 0.0 & 1 & 0.0 & 0.0 & 1 & 0.0 & 0.0 \\
\hline Japanese Encephalitis & 35 & 100.0 & 0.0 & 35 & 71.4 & 7.6 & 65.7 & 8.1 & 24 & 95.8 & 4.1 & 34 & 67.6 & 8.0 \\
\hline Marburg & 3 & 0.0 & 0.0 & 0 & - & - & - & - & 0 & - & - & 3 & 0.0 & 0.0 \\
\hline Middle East Respiratory Syndrome (MERS) & 4 & 50.0 & 25.0 & 0 & - & - & - & - & 0 & - & - & 2 & 0.0 & 0.0 \\
\hline Monkeypox & 1 & 100.0 & 0.0 & 1 & 100.0 & 0.0 & 100.0 & 0.0 & 1 & 100.0 & 0.0 & 1 & 100.0 & 0.0 \\
\hline Norovirus & 6 & 100.0 & 0.0 & 5 & 0.0 & 0.0 & 0.0 & 0.0 & 0 & - & - & 5 & 0.0 & 0.0 \\
\hline Otitis Media & 23 & 95.7 & 4.3 & 22 & 81.8 & 8.2 & 45.5 & 10.6 & 18 & 55.6 & 11.7 & 23 & 43.5 & 10.3 \\
\hline Rabies & 47 & 91.5 & 4.1 & 40 & 87.5 & 5.2 & 65.0 & 8.1 & 30 & 86.7 & 6.2 & 39 & 66.7 & 7.5 \\
\hline Respiratory Infections & 640 & 79.1 & 1.6 & 465 & 66.9 & 2.2 & 50.1 & 2.4 & 287 & 81.2 & 2.3 & 575 & 40.5 & 2.0 \\
\hline Rotavirus & 72 & 97.2 & 1.9 & 70 & 91.4 & 3.3 & 68.6 & 6.0 & 53 & 90.6 & 4.0 & 61 & 78.7 & 5.2 \\
\hline Sepsis & 13 & 38.5 & 13.5 & 5 & 80.0 & 17.9 & 0.0 & 0.0 & 4 & 0.0 & 0.0 & 13 & 0.0 & 0.0 \\
\hline Severe Acute Respiratory Syndrome (SARS) & 1 & 0.0 & 0.0 & 0 & - & - & - & - & 0 & - & - & 1 & 0.0 & 0.0 \\
\hline Smallpox & 11 & 81.8 & 11.6 & 8 & 62.5 & 17.1 & 50.0 & 17.7 & 5 & 80.0 & 17.9 & 10 & 40.0 & 15.5 \\
\hline Urinary Tract Infections & 3 & 100.0 & 0.0 & 3 & 100.0 & 0.0 & 0.0 & 0.0 & 1 & 0.0 & 0.0 & 1 & 0.0 & 0.0 \\
\hline West Nile Virus (WNV) & 4 & 25.0 & 21.7 & 1 & 100.0 & 0.0 & 0.0 & 0.0 & 1 & 0.0 & 0.0 & 4 & 0.0 & 0.0 \\
\hline Yellow Fever & 30 & 90.0 & 5.5 & 26 & 73.1 & 8.7 & 57.7 & 10.5 & 15 & 100.0 & 0.0 & 25 & 60.0 & 9.8 \\
\hline Zika & 2 & 0.0 & 0.0 & 0 & - & - & - & - & 0 & - & - & 2 & 0.0 & 0.0 \\
\hline Others & 350 & 87.1 & 1.8 & 268 & 63.4 & 2.9 & 47.0 & 3.2 & 142 & 88.7 & 2.7 & 285 & 44.2 & 2.9 \\
\hline Total & 1,838 & 82.5 & 0.9 & 1,339 & 65.4 & 1.3 & 45.9 & 1.4 & 768 & 80.1 & 1.4 & 1552 & 39.6 & 1.2 \\
\hline
\end{tabular}

In contrast, non-industry-sponsored vaccine development programs have an overall PoS of only 6.8\% (SE = 1.0\%), with $\mathrm{PoS}_{12}, \mathrm{PoS}_{23}$, and $\mathrm{PoS}_{3 \mathrm{~A}}$ estimates of $63.3 \%$ (SE $=1.8 \%$ ),

13 May 2020

(C) 2020 by Lo, Siah, and Wong

Page 9 of 25

All Rights Reserved 
$37.3 \%$ ( $\mathrm{SE}=2.6 \%$ ), and 39.8\% ( $\mathrm{SE}=4.9 \%$ ), respectively (Table 2). The top three indications with the highest overall success rates for non-industry-sponsored drug development programs are otitis media $(28.6 \%, \mathrm{SE}=17.1 \%)$, rabies $(25.0 \%, \mathrm{SE}=10.8 \%)$, and Japanese encephalitis $(25.0 \%, \mathrm{SE}=21.7 \%)$. The latter estimates are derived from only a handful of samples and must be interpreted with caution as their large standard errors suggest.

Table 2. The probabilities of success (PoSs) of non-industry-sponsored vaccine development programs. A: regulatory approval; P1: phase 1; P2: phase 2; P3: phase 3; SE = standard error.

\begin{tabular}{|c|c|c|c|c|c|c|c|c|c|c|c|c|c|c|}
\hline Disease & P1 Paths & $\mathrm{PoS}_{12}$ & SE & P2 Paths & $\mathrm{PoS}_{23}$ & SE & $\mathrm{PoS}_{2 \mathrm{~A}}$ & SE & P3 Paths & $\mathrm{PoS}_{3 \mathrm{~A}}$ & SE & Paths & $\operatorname{PoS}_{1 \mathrm{~A}}$ & SE \\
\hline Bacterial Skin Infection & 3 & 100.0 & 0.0 & 0 & - & - & - & - & 0 & - & - & 0 & 0.0 & 0.0 \\
\hline Clostridium difficile & 3 & 66.7 & 27.2 & 0 & - & - & - & - & 0 & - & - & 1 & 0.0 & 0.0 \\
\hline Cytomegalovirus Infection (CMV) & 14 & 50.0 & 13.4 & 5 & 40.0 & 21.9 & 40.0 & 21.9 & 2 & 100.0 & 0.0 & 12 & 16.7 & 10.8 \\
\hline Ebola & 8 & 12.5 & 11.7 & 0 & - & - & - & - & 0 & - & - & 7 & 0.0 & 0.0 \\
\hline Hepatitis B Virus (HBV) & 58 & 91.4 & 3.7 & 48 & 47.9 & 7.2 & 8.3 & 4.3 & 16 & 25.0 & 10.8 & 46 & 8.7 & 4.2 \\
\hline Hepatitis C Virus (HCV) & 10 & 70.0 & 14.5 & 5 & 0.0 & 0.0 & 0.0 & 0.0 & 0 & - & - & 8 & 0.0 & 0.0 \\
\hline HIV & 144 & 48.6 & 4.2 & 62 & 3.2 & 2.2 & 0.0 & 0.0 & 2 & 0.0 & 0.0 & 136 & 0.0 & 0.0 \\
\hline Human Papillomavirus (HPV) & 32 & 87.5 & 5.8 & 16 & 56.3 & 12.4 & 6.3 & 6.5 & 7 & 14.3 & 13.2 & 18 & 5.6 & 5.4 \\
\hline Intra-abdominal Infections & 3 & 100.0 & 0.0 & 0 & - & - & - & - & 0 & - & - & 0 & 0.0 & 0.0 \\
\hline Japanese Encephalitis & 4 & 100.0 & 0.0 & 4 & 100.0 & 0.0 & 25.0 & 21.7 & 4 & 25.0 & 21.7 & 4 & 25.0 & 21.7 \\
\hline Middle East Respiratory Syndrome (MERS) & 1 & 100.0 & 0.0 & 0 & - & - & - & - & 0 & - & - & 0 & 0.0 & 0.0 \\
\hline Otitis Media & 7 & 100.0 & 0.0 & 7 & 28.6 & 17.1 & 28.6 & 17.1 & 2 & 100.0 & 0.0 & 7 & 28.6 & 17.1 \\
\hline Rabies & 16 & 81.3 & 9.8 & 13 & 53.8 & 13.8 & 30.8 & 12.8 & 7 & 57.1 & 18.7 & 16 & 25.0 & 10.8 \\
\hline Respiratory Infections & 175 & 66.9 & 3.6 & 101 & 51.5 & 5.0 & 16.8 & 3.9 & 41 & 41.5 & 7.7 & 148 & 11.5 & 2.6 \\
\hline Rotavirus & 5 & 80.0 & 17.9 & 4 & 50.0 & 25.0 & 0.0 & 0.0 & 1 & 0.0 & 0.0 & 4 & 0.0 & 0.0 \\
\hline Sepsis & 7 & 42.9 & 18.7 & 2 & 0.0 & 0.0 & 0.0 & 0.0 & 0 & - & - & 6 & 0.0 & 0.0 \\
\hline Severe Acute Respiratory Syndrome (SARS) & 1 & 0.0 & 0.0 & 0 & - & - & - & - & 0 & - & - & 1 & 0.0 & 0.0 \\
\hline Smallpox & 11 & 63.6 & 14.5 & 6 & 16.7 & 15.2 & 16.7 & 15.2 & 1 & 100.0 & 0.0 & 10 & 10.0 & 9.5 \\
\hline Urinary Tract Infections & 3 & 100.0 & 0.0 & 1 & 0.0 & 0.0 & 0.0 & 0.0 & 0 & - & - & 1 & 0.0 & 0.0 \\
\hline West Nile Virus (WNV) & 4 & 0.0 & 0.0 & 0 & - & - & - & - & 0 & - & - & 4 & 0.0 & 0.0 \\
\hline Yellow Fever & 9 & 66.7 & 15.7 & 6 & 33.3 & 19.2 & 0.0 & 0.0 & 1 & 0.0 & 0.0 & 8 & 0.0 & 0.0 \\
\hline Zika & 5 & 40.0 & 21.9 & 0 & - & - & - & - & 0 & - & - & 3 & 0.0 & 0.0 \\
\hline Others & 183 & 57.9 & 3.6 & 71 & 35.2 & 5.7 & 9.9 & 3.8 & 14 & 50.0 & 13.4 & 137 & 5.1 & 1.9 \\
\hline Total & 706 & 63.3 & 1.8 & 351 & 37.3 & 2.6 & 11.1 & 1.8 & 98 & 39.8 & 4.9 & 577 & 6.8 & 1.0 \\
\hline
\end{tabular}

\subsection{Nonvaccine Anti-Infective Therapeutics}

In contrast to vaccines, which are intended to prevent disease, a number of alternatives have been developed to treat-and, in some cases, cure-patients afflicted with an infectious disease. According to our data set, 3,851 and 2,978 industry-sponsored and non-industry-sponsored nonvaccine drug development programs, respectively, have been initiated in the area of infectious disease (see Figure 5). The top three diseases with the greatest number of industry-sponsored drug development programs are respiratory infections $(21.8 \%)$, HIV (15.7\%) and hepatitis C virus, or HCV (14.1\%). Together, they comprise $51.6 \%$ of all industry-sponsored nonvaccine development programs. Non-industry anti-infectious-disease drug development programs focus on treating respiratory infections (20.5\%), HIV (13.9\%), and bacterial skin infection (12.1\%).

With respect to addressing the most recent virus outbreaks-MERS, SARS, Ebola, and Zika-a total of nine industry-sponsored and 36 non-industry-sponsored nonvaccine drug

13 May 2020

(C) 2020 by Lo, Siah, and Wong

Page 10 of 25

All Rights Reserved 
development programs were initiated over the past 20 years, and there have been no approved therapies to date.

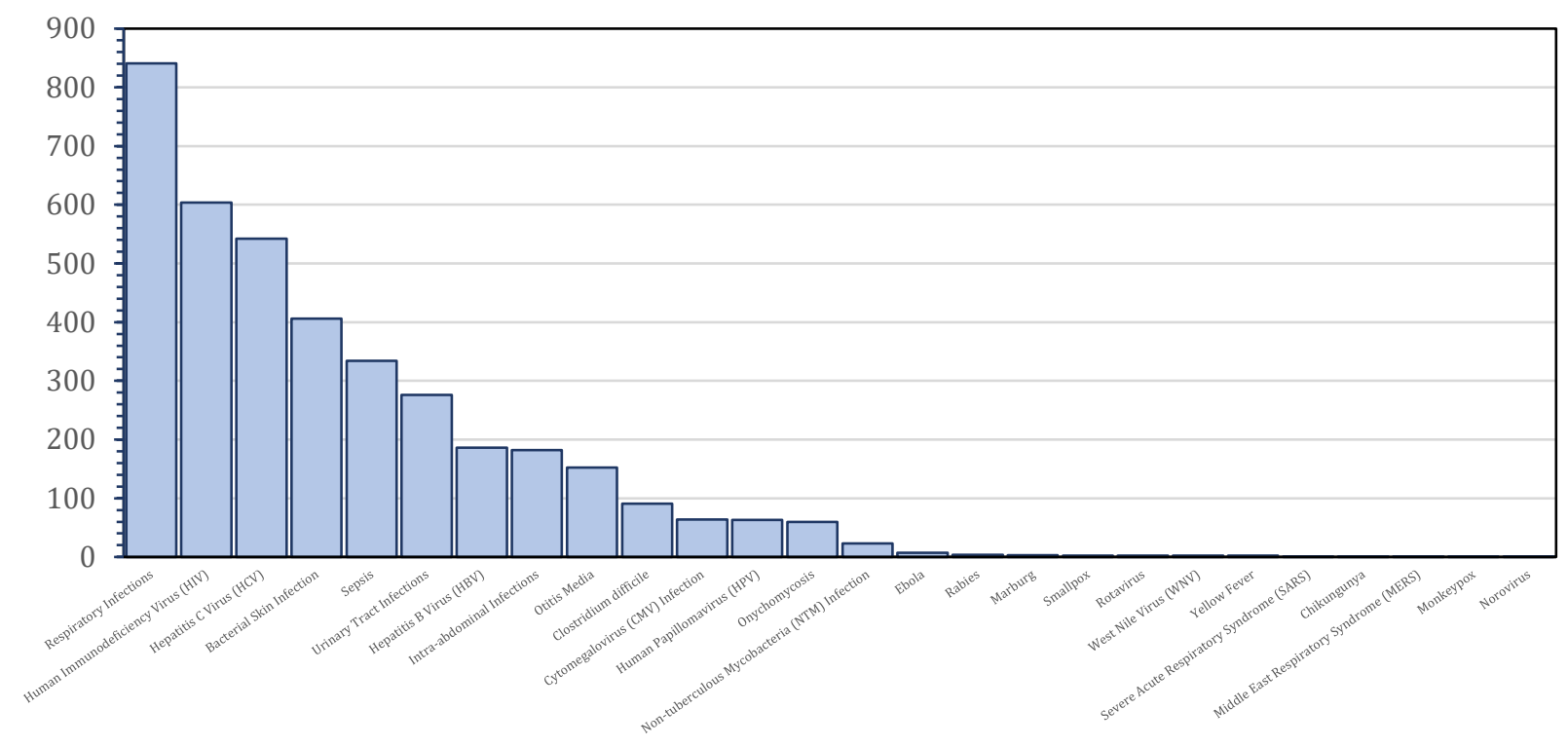

(a) Number of industry-sponsored, nonvaccine drug development programs

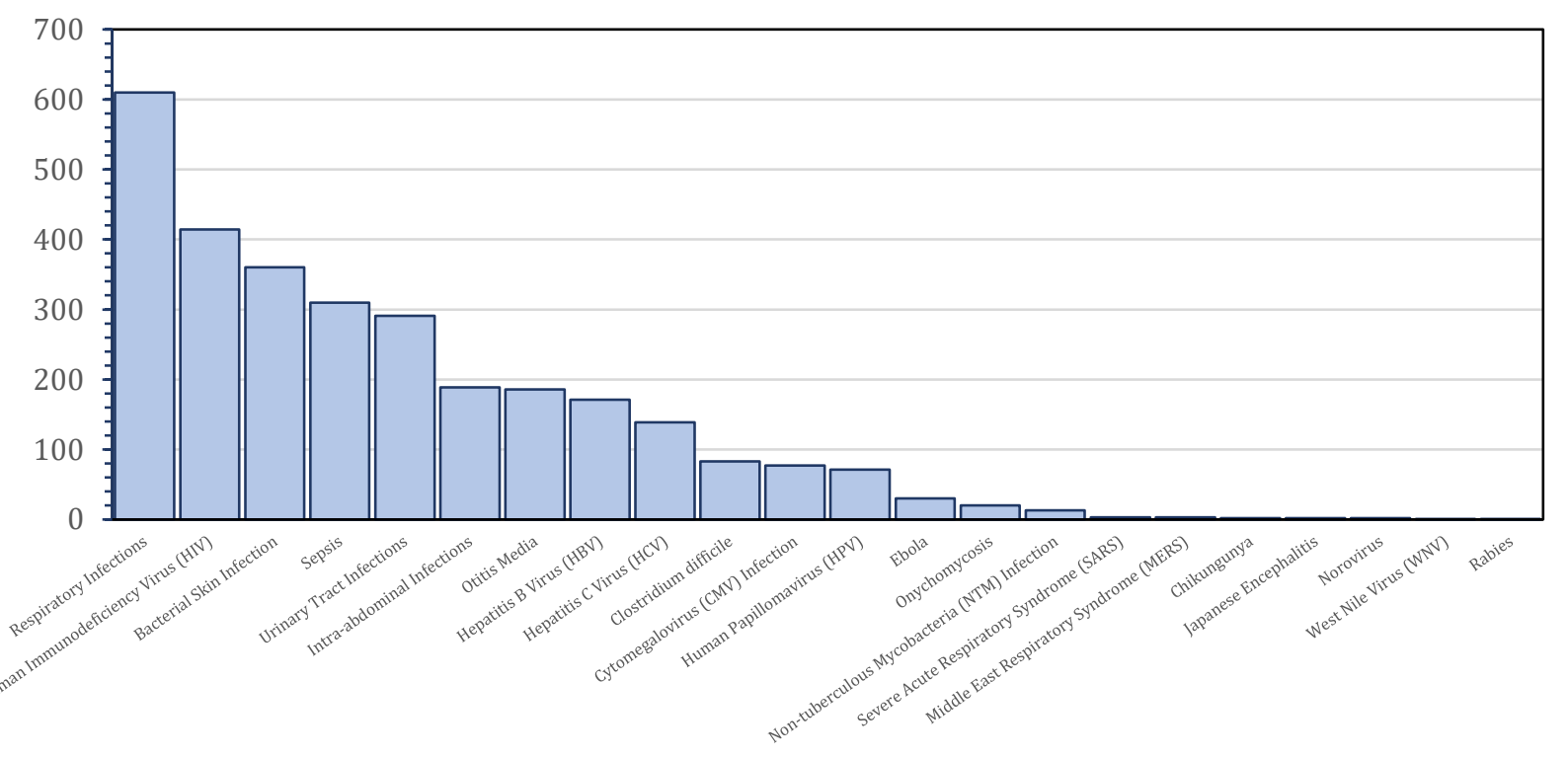

(b) Number of non-industry-sponsored, nonvaccine drug development programs

Figure 5. Number of nonvaccine drug development programs for each disease in the 'Infectious Disease' category. HBV, hepatitis B virus; HCV, hepatitis C virus; HIV, human immunodeficiency virus; HPV, human papillomavirus.

From Table 3, we can see that the overall PoS across all industry-sponsored drug development programs treating infectious diseases is $16.3 \%$ ( $\mathrm{SE}=0.7 \%$ ). The $\mathrm{PoS}_{12}, \mathrm{PoS}_{23}$, and $\mathrm{PoS}_{3 \mathrm{~A}}$ are $65.0 \%(\mathrm{SE}=0.8 \%$ ), 64.3\% (SE $=1.0 \%$ ), and 51.1\% (SE = 1.6\%), respectively. Based on our data, the highest success rates for industry-sponsored nonvaccine 
development programs have been for smallpox (100.0\%, SE $=0.0 \%$ ), cytomegalovirus (CMV) infection $(31.8 \%, \mathrm{SE}=7.0 \%)$, and onychomycosis $(29.8 \%$, SE $=6.7 \%)$. There are currently no nonvaccine therapies approved for rotavirus, SARS, rabies, Ebola, West Nile virus, Marburg, yellow fever, chikungunya, MERS, monkeypox, or norovirus. With the exception of norovirus and MERS, these diseases without any vaccine are predominantly prevalent in nonindustrialized nations, and thus represent neglected diseases. It is also interesting that for the latter eight diseases, even the $\mathrm{PoS}_{12}$ is low. Since phase 1 trials in the development of anti-infective therapies focus primarily on safety, understanding the pharmacokinetics of the compound, and maximum tolerable dose levels, it can be inferred that the drugs tested are either of high toxicity or lack the necessary characteristics required for optimal absorption, distribution, metabolism, and excretion (ODME), or perhaps failed to advance due to financial constraints.

Table 3. The probabilities of success (PoSs) of industry-sponsored, nonvaccine anti-infective drug development programs for the treatment of infectious diseases. A: regulatory approval; P1: phase 1; P2: phase 2; P3: phase 3; SE = standard error.

\begin{tabular}{|c|c|c|c|c|c|c|c|c|c|c|c|c|c|c|}
\hline Disease & P1 Paths & $\mathrm{PoS}_{12}$ & SE & P2 Paths & $\mathrm{PoS}_{23}$ & SE & $\mathrm{PoS}_{2 \mathrm{~A}}$ & SE & P3 Paths & $\mathrm{PoS}_{3 \mathrm{~A}}$ & SE & Paths & $\mathrm{PoS}_{1 \mathrm{~A}}$ & SE \\
\hline Bacterial Skin Infection & 406 & 54.9 & 2.5 & 207 & 72.9 & 3.1 & 19.8 & 3.2 & 104 & 39.4 & 4.8 & 343 & 12.0 & 1.8 \\
\hline Chikungunya & 1 & 0.0 & 0.0 & 0 & - & - & - & - & 0 & - & - & 1 & 0.0 & 0.0 \\
\hline Clostridium difficile & 91 & 83.5 & 3.9 & 66 & 53.0 & 6.1 & 4.5 & 2.8 & 25 & 12.0 & 6.5 & 71 & 4.2 & 2.4 \\
\hline Cytomegalovirus Infection (CMV) & 64 & 87.5 & 4.1 & 43 & 60.5 & 7.5 & 32.6 & 7.8 & 19 & 73.7 & 10.1 & 44 & 31.8 & 7.0 \\
\hline Ebola & 7 & 28.6 & 17.1 & 1 & 0.0 & 0.0 & 0.0 & 0.0 & 0 & - & - & 6 & 0.0 & 0.0 \\
\hline Hepatitis B Virus (HBV) & 186 & 77.4 & 3.1 & 105 & 68.6 & 4.5 & 36.2 & 5.2 & 54 & 70.4 & 6.2 & 129 & 29.5 & 4.0 \\
\hline Hepatitis C Virus (HCV) & 542 & 68.8 & 2.0 & 348 & 52.3 & 2.7 & 23.6 & 2.4 & 155 & 52.9 & 4.0 & 490 & 16.7 & 1.7 \\
\hline Human Immunodeficiency Virus (HIV) & 604 & 63.2 & 2.0 & 326 & 59.8 & 2.7 & 39.3 & 2.8 & 167 & 76.6 & 3.3 & 520 & 24.6 & 1.9 \\
\hline Human Papillomavirus (HPV) & 63 & 85.7 & 4.4 & 34 & 23.5 & 7.3 & 11.8 & 5.7 & 6 & 66.7 & 19.2 & 41 & 9.8 & 4.6 \\
\hline Intra-abdominal Infections & 182 & 68.7 & 3.4 & 113 & 72.6 & 4.2 & 2.7 & 2.0 & 35 & 8.6 & 4.7 & 123 & 2.4 & 1.4 \\
\hline Marburg & 3 & 0.0 & 0.0 & 0 & - & - & - & - & 0 & - & - & 3 & 0.0 & 0.0 \\
\hline Middle East Respiratory Syndrome (MERS) & 1 & 0.0 & 0.0 & 0 & - & - & - & - & 0 & - & - & 1 & 0.0 & 0.0 \\
\hline Monkeypox & 1 & 0.0 & 0.0 & 0 & - & - & - & - & 0 & - & - & 1 & 0.0 & 0.0 \\
\hline Non-tuberculous Mycobacteria (NTM) Infection & 23 & 87.0 & 7.0 & 16 & 62.5 & 12.1 & 6.3 & 7.7 & 4 & 25.0 & 21.7 & 13 & 7.7 & 7.4 \\
\hline Norovirus & 1 & 0.0 & 0.0 & 0 & - & - & - & - & 0 & - & - & 1 & 0.0 & 0.0 \\
\hline Onychomycosis & 60 & 85.0 & 4.6 & 44 & 63.6 & 7.3 & 31.8 & 7.6 & 22 & 63.6 & 10.3 & 47 & 29.8 & 6.7 \\
\hline Otitis Media & 152 & 48.0 & 4.1 & 68 & 80.9 & 4.8 & 51.5 & 6.2 & 51 & 68.6 & 6.5 & 143 & 24.5 & 3.6 \\
\hline Rabies & 4 & 75.0 & 21.7 & 0 & - & - & - & - & 0 & - & - & 1 & 0.0 & 0.0 \\
\hline Respiratory Infections & 841 & 64.2 & 1.7 & 476 & 70.0 & 2.1 & 22.9 & 2.2 & 222 & 49.1 & 3.4 & 666 & 16.4 & 1.4 \\
\hline Rotavirus & 2 & 100.0 & 0.0 & 2 & 0.0 & 0.0 & 0.0 & 0.0 & 0 & - & - & 2 & 0.0 & 0.0 \\
\hline Sepsis & 334 & 66.8 & 2.6 & 206 & 64.6 & 3.3 & 10.2 & 2.4 & 81 & 25.9 & 4.9 & 265 & 7.9 & 1.7 \\
\hline Severe Acute Respiratory Syndrome (SARS) & 1 & 100.0 & 0.0 & 1 & 0.0 & 0.0 & 0.0 & 0.0 & 0 & - & - & 1 & 0.0 & 0.0 \\
\hline Smallpox & 2 & 100.0 & 0.0 & 2 & 100.0 & 0.0 & 100.0 & 0.0 & 2 & 100.0 & 0.0 & 2 & 100.0 & 0.0 \\
\hline Urinary Tract Infections & 276 & 55.1 & 3.0 & 143 & 72.0 & 3.8 & 10.5 & 3.2 & 51 & 29.4 & 6.4 & 215 & 7.0 & 1.7 \\
\hline West Nile Virus (WNV) & 2 & 50.0 & 35.4 & 1 & 0.0 & 0.0 & 0.0 & 0.0 & 0 & - & - & 2 & 0.0 & 0.0 \\
\hline Yellow Fever & 2 & 0.0 & 0.0 & 0 & - & - & - & - & 0 & - & - & 2 & 0.0 & 0.0 \\
\hline Total & 3,851 & 65.0 & 0.8 & 2,202 & 64.3 & 1.0 & 23.2 & 1.0 & 998 & 51.1 & 1.6 & 3133 & 16.3 & 0.7 \\
\hline
\end{tabular}

For non-industry-sponsored nonvaccine development programs, the overall PoS is 8.2\% (SE $=0.6 \%$ ) while $\mathrm{PoS}_{12}, \mathrm{PoS}_{23}$, and $\mathrm{PoS}_{3 \mathrm{~A}}$ are $61.0 \%$ (SE = 0.9\%), 65.2\% (SE = 1.2\%), and $30.0 \%$ (SE $=1.8 \%$ ), respectively (see Table 4). The top three indications with the highest overall success rates for non-industry-sponsored nonvaccine development programs are CMV infection $(23.5 \%, \mathrm{SE}=5.9 \%)$, clostridium difficile $(20.5 \%, \mathrm{SE}=6.5 \%)$, and sepsis $(17.4 \%, \mathrm{SE}=2.6 \%)$.

13 May 2020

(C) 2020 by Lo, Siah, and Wong

Page 12 of 25

All Rights Reserved 
Table 4. The probabilities of success (PoSs) of non-industry-sponsored, nonvaccine anti-infective drug development programs. A: regulatory approval; P1: phase 1; P2: phase 2; P3: phase 3; SE = standard error.

\begin{tabular}{|c|c|c|c|c|c|c|c|c|c|c|c|c|c|c|}
\hline Disease & P1 Paths & $\mathrm{PoS}_{12}$ & SE & P2 Paths & $\mathrm{PoS}_{23}$ & SE & $\mathrm{PoS}_{2 \mathrm{~A}}$ & SE & P3 Paths & $\operatorname{PoS}_{3 \mathrm{~A}}$ & SE & Paths & $\operatorname{PoS}_{1 \mathrm{~A}}$ & SE \\
\hline Bacterial Skin Infection & 360 & 46.4 & 2.6 & 151 & 81.5 & 3.2 & 19.2 & 3.7 & 85 & 34.1 & 5.1 & 306 & 9.5 & 1.7 \\
\hline Chikungunya & 2 & 100.0 & 0.0 & 2 & 50.0 & 35.4 & 0.0 & 0.0 & 1 & 0.0 & 0.0 & 2 & 0.0 & 0.0 \\
\hline Clostridium difficile & 83 & 94.0 & 2.6 & 51 & 76.5 & 5.9 & 15.7 & 6.2 & 22 & 36.4 & 10.3 & 39 & 20.5 & 6.5 \\
\hline Cytomegalovirus Infection (CMV) & 77 & 83.1 & 4.3 & 51 & 51.0 & 7.0 & 23.5 & 6.9 & 13 & 92.3 & 7.4 & 51 & 23.5 & 5.9 \\
\hline Ebola & 30 & 96.7 & 3.3 & 28 & 14.3 & 6.6 & 0.0 & 0.0 & 2 & 0.0 & 0.0 & 27 & 0.0 & 0.0 \\
\hline Hepatitis B Virus (HBV) & 171 & 49.1 & 3.8 & 73 & 47.9 & 5.8 & 1.4 & 1.4 & 31 & 3.2 & 3.2 & 156 & 0.6 & 0.6 \\
\hline Hepatits C Virus (HCV) & 139 & 84.2 & 3.1 & 112 & 43.8 & 4.7 & 8.9 & 2.9 & 33 & 30.3 & 8.0 & 118 & 8.5 & 2.6 \\
\hline Human Immunodeficiency Virus (HIV) & 414 & 61.1 & 2.4 & 195 & 49.2 & 3.6 & 13.3 & 2.6 & 75 & 34.7 & 5.5 & 335 & 7.8 & 1.5 \\
\hline Human Papillomavirus (HPV) & 71 & 88.7 & 3.8 & 42 & 42.9 & 7.6 & 2.4 & 2.7 & 8 & 12.5 & 11.7 & 40 & 2.5 & 2.5 \\
\hline Intra-abdominal Infections & 189 & 66.1 & 3.4 & 112 & 76.8 & 4.0 & 12.5 & 3.8 & 51 & 27.5 & 6.2 & 141 & 9.9 & 2.5 \\
\hline Japanese Encephalitis & 2 & 100.0 & 0.0 & 2 & 0.0 & 0.0 & 0.0 & 0.0 & 0 & - & - & 2 & 0.0 & 0.0 \\
\hline Middle East Respiratory Syndrome (MERS) & 3 & 100.0 & 0.0 & 3 & 66.7 & 27.2 & 0.0 & 0.0 & 0 & - & - & 1 & 0.0 & 0.0 \\
\hline Non-tuberculous Mycobacteria (NTM) Infection & 13 & 84.6 & 10.0 & 9 & 44.4 & 16.6 & 11.1 & 11.9 & 2 & 50.0 & 35.4 & 9 & 11.1 & 10.5 \\
\hline Norovirus & 2 & 100.0 & 0.0 & 1 & 0.0 & 0.0 & 0.0 & 0.0 & 0 & - & - & 1 & 0.0 & 0.0 \\
\hline Onychomycosis & 20 & 75.0 & 9.7 & 15 & 66.7 & 12.2 & 0.0 & 0.0 & 6 & 0.0 & 0.0 & 16 & 0.0 & 0.0 \\
\hline Otitis Media & 186 & 30.1 & 3.4 & 53 & 56.6 & 6.8 & 7.5 & 3.9 & 24 & 16.7 & 7.6 & 177 & 2.3 & 1.1 \\
\hline Rabies & 1 & 0.0 & 0.0 & 0 & - & - & - & - & 0 & - & - & 1 & 0.0 & 0.0 \\
\hline Respiratory Infections & 610 & 58.5 & 2.0 & 323 & 72.8 & 2.5 & 11.5 & 2.1 & 141 & 26.2 & 3.7 & 482 & 7.7 & 1.2 \\
\hline Sepsis & 310 & 80.0 & 2.3 & 227 & 77.5 & 2.8 & 15.9 & 3.0 & 94 & 38.3 & 5.0 & 207 & 17.4 & 2.6 \\
\hline Severe Acute Respiratory Syndrome (SARS) & 3 & 100.0 & 0.0 & 3 & 100.0 & 0.0 & 0.0 & 0.0 & 2 & 0.0 & 0.0 & 2 & 0.0 & 0.0 \\
\hline Urinary Tract Infections & 291 & 46.7 & 2.9 & 126 & 73.8 & 3.9 & 10.3 & 3.4 & 49 & 26.5 & 6.3 & 237 & 5.5 & 1.5 \\
\hline West Nile Virus (WNV) & 1 & 100.0 & 0.0 & 1 & 0.0 & 0.0 & 0.0 & 0.0 & 0 & - & - & 1 & 0.0 & 0.0 \\
\hline Total & 2,978 & 61.0 & 0.9 & 1,580 & 65.2 & 1.2 & 12.2 & 0.9 & 639 & 30.0 & 1.8 & 2351 & 8.2 & 0.6 \\
\hline
\end{tabular}

\section{Industry-Sponsored Development Programs}

In an attempt to shed more light on the industry-sponsored vaccine and nonvaccine drug development programs, we classify the diseases by their biological family and transmission type. The classifications are presented in Table 9 in the Appendix. We then compute the PoSs using these classifications.

Looking at the vaccine PoSs by transmission route (see Table 5), we see that vaccines for diseases transmitted through animal bites have the highest overall PoS $(61.3 \%$, SE = 4.7\%), whereas no vaccine has been developed for diseases transmitted through contaminated food or water. 
Table 5. The probabilities of success (PoSs) of industry-sponsored vaccine development programs, grouped by the transmission route. A: regulatory approval; P1: phase 1; P2: phase 2; P3: phase 3; SE = standard error.

\begin{tabular}{|c|c|c|c|c|c|c|c|c|c|c|c|c|c|c|}
\hline Disease & P1 Paths & $\mathrm{PoS}_{12}$ & SE & P2 Paths & $\mathrm{PoS}_{23}$ & SE & $\mathrm{PoS}_{2 \mathrm{~A}}$ & SE & P3 Paths & $\mathrm{PoS}_{3 \mathrm{~A}}$ & SE & Paths & $\mathrm{PoS}_{1 \mathrm{~A}}$ & SE \\
\hline Animal bites & 125 & 89.6 & 2.7 & 103 & 78.6 & 4.0 & 63.1 & 5.0 & 71 & 91.5 & 3.3 & 106 & 61.3 & 4.7 \\
\hline Contaminated food or water & 6 & 100.0 & 0.0 & 5 & 0.0 & 0.0 & 0.0 & 0.0 & 0 & - & - & 5 & 0.0 & 0.0 \\
\hline Human-human (Others) & 643 & 82.4 & 1.5 & 446 & 62.8 & 2.3 & 39.7 & 2.4 & 238 & 74.4 & 2.8 & 517 & 34.2 & 2.1 \\
\hline Human-human (Airborne) & 16 & 68.8 & 11.6 & 8 & 62.5 & 17.1 & 50.0 & 17.7 & 5 & 80.0 & 17.9 & 13 & 30.8 & 12.8 \\
\hline Multiple or others & 1,048 & 81.9 & 1.2 & 777 & 65.6 & 1.7 & 47.5 & 1.9 & 454 & 81.3 & 1.8 & 911 & 40.5 & 1.6 \\
\hline Total & 1,838 & 82.5 & 0.9 & 1,339 & 65.4 & 1.3 & 45.9 & 1.4 & 768 & 80.1 & 1.4 & 1552 & 39.6 & 1.2 \\
\hline
\end{tabular}

We find that companies have been most successful in developing nonvaccine treatments for diseases transmitted between humans through the air, with $50.0 \%$ (SE = $25.0 \%$ ) of all drug development programs making it from phase 1 to regulatory approval (see Table 6). Unfortunately, this is based on only four drug development programs and may not be indicative of the general trend. Treatments for diseases that transmit through 'human to human (others)' have an overall PoS of $21.5 \%$ (SE $=1.2 \%$ ) while no approval is observed for diseases transmitted through 'animal bites' or 'contaminated food or water'.

Table 6. The probabilities of success (PoSs) of industry-sponsored, nonvaccine anti-infective drug development programs, grouped by the transmission route. A: regulatory approval; P1: phase 1; P2: phase 2; P3: phase 3; SE = standard error .

\begin{tabular}{|c|c|c|c|c|c|c|c|c|c|c|c|c|c|c|}
\hline Disease & P1 Paths & $\mathrm{PoS}_{12}$ & SE & P2 Paths & $\mathrm{PoS}_{23}$ & SE & $\mathrm{PoS}_{2 \mathrm{~A}}$ & SE & P3 Paths & $\mathrm{PoS}_{3 \mathrm{~A}}$ & SE & Paths & $\mathrm{PoS}_{1 \mathrm{~A}}$ & SE \\
\hline Animal bites & 10 & 40.0 & 15.5 & 1 & 0.0 & 0.0 & 0.0 & 0.0 & 0 & - & - & 7 & 0.0 & 0.0 \\
\hline Contaminated food or water & 1 & 0.0 & 0.0 & 0 & - & - & - & - & 0 & - & - & 1 & 0.0 & 0.0 \\
\hline Human-human (Others) & 1,471 & 68.9 & 1.2 & 859 & 56.2 & 1.7 & 31.0 & 1.7 & 401 & 66.3 & 2.4 & 1235 & 21.5 & 1.2 \\
\hline Human-human (Airborne) & 4 & 75.0 & 21.7 & 3 & 66.7 & 27.2 & 66.7 & 27.2 & 2 & 100.0 & 0.0 & 4 & 50.0 & 25.0 \\
\hline Multiple or others & 2,365 & 62.7 & 1.0 & 1,339 & 69.5 & 1.3 & 18.1 & 1.2 & 595 & 40.7 & 2.0 & 1886 & 12.8 & 0.8 \\
\hline Total & 3,851 & 65.0 & 0.8 & 2,202 & 64.3 & 1.0 & 23.2 & 1.0 & 998 & 51.1 & 1.6 & 3133 & 16.3 & 0.7 \\
\hline
\end{tabular}

When we classify the vaccines by the biological family of the infectious agent (Table 7), we see that reoviridae (e.g., rotavirus), rhabdoviridae (e.g., rabies), and hepadnaviridae (e.g., HBV) are the three biological families with the highest overall PoSs for vaccines at 78.7\%, ( $S E=5.2 \%), 66.7 \%$ ( $S E=7.5 \%$ ), and 53.7\% ( $S E=3.6 \%)$, respectively. We have yet to see a vaccine for diseases caused by agents in the biological families of retroviridae (e.g., HIV), caliciviridae (e.g., norovirus), clostridiaceae (e.g., clostridium difficile), coronaviridae (e.g., SARS, MERS), herpesviridae (e.g., CMV infection), or togaviridae (e.g., chikungunya). 
Table 7. The probabilities of success (PoSs) of industry-sponsored vaccine development programs, grouped by the biological family. A: regulatory approval; P1: phase 1; P2: phase 2; P3: phase 3; SE = standard error.

\begin{tabular}{|c|c|c|c|c|c|c|c|c|c|c|c|c|c|c|}
\hline Disease & P1 Paths & $\mathrm{PoS}_{12}$ & SE & P2 Paths & $\mathrm{PoS}_{23}$ & SE & $\mathbf{P o S}_{2 \mathrm{~A}}$ & SE & P3 Paths & $\mathbf{P o S}_{3 \mathrm{~A}}$ & SE & Paths & $\mathbf{P o S}_{1 \mathrm{~A}}$ & SE \\
\hline Caliciviridae & 6 & 100.0 & 0.0 & 5 & 0.0 & 0.0 & 0.0 & 0.0 & 0 & - & - & 5 & 0.0 & 0.0 \\
\hline Clostridiaceae & 6 & 100.0 & 0.0 & 6 & 33.3 & 19.2 & 0.0 & 0.0 & 0 & - & - & 4 & 0.0 & 0.0 \\
\hline Coronaviridae & 5 & 40.0 & 21.9 & 0 & - & - & - & - & 0 & - & - & 3 & 0.0 & 0.0 \\
\hline & 16 & 43.8 & 12.4 & 7 & 57.1 & 18.7 & 28.6 & 20.2 & 2 & 100.0 & 0.0 & 14 & 14.3 & 9.4 \\
\hline & & & 2.3 & & & 4.1 & & 4.3 & 70 & & 3.6 & 165 & 38.2 & 3.8 \\
\hline Hepadnaviridae & & 94.8 & 1.5 & 187 & 74.9 & 3.2 & 54.5 & 3.7 & 132 & 77.3 & 3.6 & 190 & 53.7 & 3.6 \\
\hline Herpesviridae & 14 & 57.1 & 13.2 & 3 & 33.3 & 27.2 & 0.0 & 0.0 & 0 & - & - & 8 & 0.0 & 0.0 \\
\hline Multiple or others & 1,042 & 81.8 & 1.2 & 771 & 65.9 & 1.7 & 47.9 & 1.9 & 454 & 81.3 & 1.8 & 907 & 40.7 & 1.6 \\
\hline Poxviridae & 12 & 83.3 & 10.8 & 9 & 66.7 & 15.7 & 55.6 & 16.6 & 6 & 83.3 & 15.2 & 11 & 45.5 & 15.0 \\
\hline Reoviridae & 72 & 97.2 & 1.9 & 70 & 91.4 & 3.3 & 68.6 & 6.0 & 53 & 90.6 & 4.0 & 61 & 78.7 & 5.2 \\
\hline Retroviridae & 181 & 65.2 & 3.5 & 95 & 36.8 & 4.9 & 0.0 & 0.0 & 21 & 0.0 & 0.0 & 144 & 0.0 & 0.0 \\
\hline Rhabdoviridae & 47 & 91.5 & 4.1 & 40 & 87.5 & 5.2 & 65.0 & 8.1 & 30 & 86.7 & 6.2 & 39 & 66.7 & 7.5 \\
\hline Togaviridae & 6 & 83.3 & 15.2 & 0 & - & - & - & - & 0 & - & - & 1 & 0.0 & 0.0 \\
\hline Total & 1,838 & 82.5 & 0.9 & 1,339 & 65.4 & 1.3 & 45.9 & 1.4 & 768 & 80.1 & 1.4 & 1552 & 39.6 & 1.2 \\
\hline
\end{tabular}

When we consider nonvaccine PoSs by biological family of the infectious agent (see Table 8), we see that nonvaccine therapies for poxviridae (e.g., smallpox), herpesviridae (e.g., CMV infection), and hepadnaviridae (e.g., HBV) have the highest overall PoS at 66.7\% (SE = $27.2 \%$ ), 31.8\% ( $\mathrm{SE}=7.0 \%$ ), and 29.5\% ( $\mathrm{SE}=4.0 \%$ ), respectively. For viruses in the reoviridae (e.g., rotavirus), coronaviridae (e.g., SARS, MERS), caliciviridae (e.g., norovirus), rhabdoviridae (e.g., rabies), and togaviridae (e.g., chikungunya) families, there have been less than five development programs each, and no approved treatment.

Table 8. The probabilities of success (PoSs) of industry-sponsored, nonvaccine anti-infective drug development programs, grouped by the biological family. A: regulatory approval; P1: phase 1; P2: phase 2; P3: phase 3; SE = standard error.

\begin{tabular}{|c|c|c|c|c|c|c|c|c|c|c|c|c|c|c|}
\hline Disease & P1 Paths & $\mathrm{PoS}_{12}$ & SE & P2 Paths & $\mathrm{PoS}_{23}$ & SE & $\mathrm{PoS}_{2 \mathrm{~A}}$ & SE & P3 Paths & $\mathrm{PoS}_{3 \mathrm{~A}}$ & SE & Paths & $\mathrm{PoS}_{1 \mathrm{~A}}$ & SE \\
\hline Caliciviridae & 1 & 0.0 & 0.0 & 0 & - & - & - & - & 0 & - & - & 1 & 0.0 & 0.0 \\
\hline Clostridiaceae & 91 & 83.5 & 3.9 & 66 & 53.0 & 6.1 & 4.5 & 2.8 & 25 & 12.0 & 6.5 & 71 & 4.2 & 2.4 \\
\hline Coronaviridae & 2 & 50.0 & 35.4 & 1 & 0.0 & 0.0 & 0.0 & 0.0 & 0 & - & - & 2 & 0.0 & 0.0 \\
\hline Filoviridae & 10 & 20.0 & 12.6 & 1 & 0.0 & 0.0 & 0.0 & 0.0 & 0 & - & - & 9 & 0.0 & 0.0 \\
\hline Flaviviridae & 609 & 70.3 & 1.9 & 383 & 49.6 & 2.6 & 22.5 & 2.2 & 161 & 53.4 & 3.9 & 535 & 16.1 & 1.6 \\
\hline Hepadnaviridae & 186 & 77.4 & 3.1 & 105 & 68.6 & 4.5 & 36.2 & 5.2 & 54 & 70.4 & 6.2 & 129 & 29.5 & 4.0 \\
\hline Herpesviridae & 64 & 87.5 & 4.1 & 43 & 60.5 & 7.5 & 32.6 & 7.8 & 19 & 73.7 & 10.1 & 44 & 31.8 & 7.0 \\
\hline Poxviridae & 3 & 66.7 & 27.2 & 2 & 100.0 & 0.0 & 100.0 & 0.0 & 2 & 100.0 & 0.0 & 1815 & 66.7 & 27.2 \\
\hline Reoviridae & 2 & 100.0 & 0.0 & 2 & 0.0 & 0.0 & 0.0 & 0.0 & 0 & - & - & 3 & 0.0 & 0.0 \\
\hline Retroviridae & 604 & 63.2 & 2.0 & 326 & 59.8 & 2.7 & 39.3 & 2.8 & 167 & 76.6 & 3.3 & 2 & 24.6 & 1.9 \\
\hline Rhabdoviridae & 4 & 75.0 & 21.7 & 0 & - & - & - & - & 0 & - & - & 520 & 0.0 & 0.0 \\
\hline Togaviridae & 1 & 0.0 & 0.0 & 0 & - & - & - & - & 0 & - & - & 1 & 0.0 & 0.0 \\
\hline Multiple or others & 2,274 & 61.9 & 1.0 & 1,273 & 70.3 & 1.3 & 18.8 & 1.3 & 570 & 41.9 & 2.1 & 1 & 13.2 & 0.8 \\
\hline Total & 3,851 & 65.0 & 0.8 & 2,202 & 64.3 & 1.0 & 23.2 & 1.0 & 998 & 51.1 & 1.6 & 3133 & 16.3 & 0.7 \\
\hline
\end{tabular}




\section{Discussion}

Companies producing vaccines and other therapeutics for infectious diseases have gradually been retreating from these spaces in recent years. The number of companies producing vaccines has dwindled over the past few decades, and the top four vaccine companies now make up more than $90 \%$ of the global market (Evaluate, 2018). Similarly, the top four companies producing antiviral drugs occupy about $80 \%$ of the global market (Evaluate, 2018). Antibiotic developers such Achaogen and Melinta Therapeutics have filed for bankruptcy in the past year, while large pharmaceutical companies such as Novartis and Sanofi have withdrawn from the space (Jacobs, 2019), leading the Infectious Diseases Society of America to sound the alarm about the availability of effective antibiotics (Infectious Diseases Society of America, 2019).

It should be no surprise that investors are unwilling to invest in companies producing vaccines and treatments for infectious diseases given the economics of this market (Vu et al., 2020). These have been generally regarded as low-margin products, and they have low expected growth potential compared to chronic treatments in other therapeutic areas, such as oncology or cardiovascular diseases. For example, Merck's oncology assets are estimated to have contributed $\$ 11.8$ billion in incremental revenues from 2017 to 2020; for the same period, the incremental contribution of its vaccines portfolio is estimated to be $\$ 2.7$ billion (Trefis, 2020). And Merck is the second largest vaccine maker in the world. This lack of investment has resulted in a relatively low number of development programs for vaccines and treatments of infectious diseases; only $10.4 \%$ of all industry-sponsored drug development programs launched in the past two decades are in these areas (see Table 10 in the Appendix).

Our study indicates that the technical success rate is unlikely to be a barrier to investments in new vaccines and treatments for infectious diseases, unlike cancer drugs, where the financial risk of new $R \& D$ projects comes from the reduced chance of bringing a drug-indication pair from phase 1 to market. The overall PoS of industry-sponsored vaccines and treatments for infectious diseases are above the average for all therapeutic groups (see Table 10 in the Appendix).

It is often suggested that the fundamental issue behind this lack of investment is that the market for vaccines and treatments for infectious diseases is simply not lucrative enough. Despite the expense of research and development and the need for large-scale production (Weir \& Gruber, 2016), anti-infective disease treatments are used only occasionally, while vaccine companies face an avalanche of liability lawsuits (Hensley \& Wysocki, 2005). Furthermore, the companies are at the mercy of government pricing decisions (Hu, 2018).

Apart from financial considerations, the dearth of vaccines and other treatments for infectious diseases may be due to the lack of available subjects for testing these therapeutics, especially during non-epidemic periods. This may be alleviated by having faster preclinical

13 May 2020

(C) 2020 by Lo, Siah, and Wong

All Rights Reserved

Page 16 of 25 
and clinical pathways in cases of severe infectious diseases with no existing treatments. One such pathway is the Animal Rule (FDA Approval of New Drugs When Human Efficacy Studies Are Not Ethical or Feasible, 2019; FDA Approval of Biological Products When Human Efficacy Studies Are Not Ethical or Feasible, 2019) whereby the "FDA may grant marketing approval based on adequate and well-controlled animal efficacy studies when the results of those studies establish that the drug is reasonably likely to produce clinical benefit in humans" (FDA, 2015). This has been used to approve smallpox and monkeypox vaccines, and can be expanded for the investigation of therapeutics for other potentially deadly infectious diseases with low incidence rates, such as SARS.

Even though this pathway can expedite the development of vaccines and antiinfective treatments, it still requires considerable development time as one needs to establish the equivalence of the drug mechanism between animal models and humans. While it is desirable to hasten the development of vaccines and medical product during an epidemic, biological breakthroughs and science will ultimate drive the efficiency of our ability to fight pandemics of novel pathogens.

It remains to be seen if more non-industry-sponsored research can alleviate the issue. Our study shows that only $6.8 \%(\mathrm{SE}=1.0 \%$ ) and $8.2 \%$ (SE $=0.6 \%$ ) of non-industrysponsored vaccines and nonvaccine infectious disease development programs transition from phase 1 to approval, respectively. However, this may be a result of selection bias: promising vaccine and therapeutics initiated in non-industry settings are often pursued in conjunction with industry-sponsored sponsors, whereas commercially less promising projects are more likely to be pursued by non-profit organizations.

\section{Conclusion}

The world today has never been in greater need of effective vaccines and other antiinfectives. As the COVID-19 crisis has shown, infectious diseases still have the potential to cause a catastrophically large number of deaths and disrupt the daily lives of billions. We hope that our research into the probability of successfully developing infectious disease therapeutics will inform all the stakeholders and catalyze innovation and greater investment in this critical and underserved field. 


\section{References}

Abrantes-Metz, R. M., Adams, C. P., \& Metz, A. (2004). Pharmaceutical development phases: A duration analysis (Bureau of Economics No. 274). Federal Trade Commission. https://www.ftc.gov/reports/pharmaceutical-development-phases-duration-analysis

André, F. E. (2002). How the research-based industry approaches vaccine development and establishes priorities. Developments in Biologicals, 110, 25-29.

Davis, M. M., Butchart, A. T., Wheeler, J. R. C., Coleman, M. S., Singer, D. C., \& Freed, G. L. (2011). Failure-to-success ratios, transition probabilities and phase lengths for prophylactic vaccines versus other pharmaceuticals in the development pipeline. Vaccine, 29(51), 9414-9416. https://doi.org/10.1016/j.vaccine.2011.09.128

DiMasi, J. A., Feldman, L., Seckler, A., \& Wilson, A. (2010). Trends in risks associated with new drug development: Success rates for investigational drugs. Clinical Pharmacology \& Therapeutics, 87(3), 272-277. https://doi.org/10.1038/clpt.2009.295

DiMasi, J. A., Florez, M. I., Stergiopoulos, S., Pena, Y., Smith, Z., Wilkinson, M., \& Getz, K. A. (2020). Development times and approval success rates for drugs to treat infectious diseases. Clinical Pharmacology \& Therapeutics, 107(2), 324-332. https://doi.org/10.1002/cpt.1627

Ehreth, J. (2003). The global value of vaccination. Vaccine, 21(7-8), 596-600.

Evaluate. (2018, June 6). EvaluatePharma World Preview 2018, Outlook to 2024. https://www.evaluate.com/PharmaWorldPreview2018

Food and Drug Administration Amendments Act of 2007, H.R. 3580, 110 ${ }^{\text {th }}$ Cong. (2007).

Food and Drug Administration Approval of Biological Products When Human Efficacy Studies Are Not Ethical or Feasible, 21 C.F.R. $§ \S 90-95$ (2019).

Food and Drug Administration Approval of New Drugs When Human Efficacy Studies Are Not

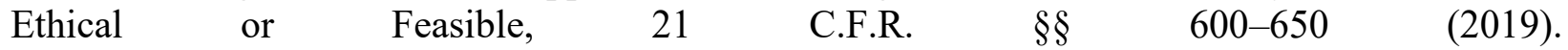
https://www.accessdata.fda.gov/scripts/cdrh/cfdocs/cfcfr/CFRSearch.cfm?CFRPart=314\&showF $\underline{\mathrm{R}=1 \& \text { subpartNode }=21: 5 \cdot 0.1 \cdot 1.4 .9}$

Food and Drug Administration [FDA]. (2015, October). Guidance for industry product development animal rule. https://www.fda.gov/files/drugs/published/Product-DevelopmentUnder-the-Animal-Rule.pdf

Hay, M., Thomas, D. W., Craighead, J. L., Economides, C., \& Rosenthal, J. (2014). Clinical development success rates for investigational drugs. Nature Biotechnology, 32(1), 40-51. https://doi.org/10.1038/nbt.2786 
Hensley, S., \& Wysocki Jr., B. (2005, November 8). As industry profits elsewhere, U.S. lacks vaccines, antibiotics. The Wall Street Journal. https://www.wsj.com/articles/SB113141787830190837

Hu, C. (2018, July 21). Major pharmaceutical companies like Novartis dropping antibiotics projects and future tools against superbugs. Business Insider. https://www.businessinsider.com/major-pharmaceutical-companies-dropping-antibiotic-projectssuperbugs-2018-7

Infectious Diseases Society of America. (2019, December 27). CORRECTION: Antibiotic company starts bankruptcy proceedings; Highlights urgent need for investment in infection fighting drugs. https://www.idsociety.org/news--publications-new/articles/2019/CORRECTIONAntibiotic-Company-Starts-Bankruptcy-Proceedings-Highlights-Urgent-Need-for-Investment-inInfection-Fighting-Drugs/

Jacobs, A. (2019, December 25). Crisis looms in antibiotics as drug makers go bankrupt. The New York Times. https://www.nytimes.com/2019/12/25/health/antibiotics-new-resistance.html

Kieny, M. P., \& Girard, M. P. (2005). Human vaccine research and development: An overview. Vaccine, 23(50), 5705-5707. https://doi.org/10.1016/j.vaccine.2005.07.077

Langreth, R. (2019, May 3). Antibiotics aren't profitable enough for big pharma to make more. Bloomberg Businessweek. https://www.bloomberg.com/news/articles/2019-05-03/antibioticsaren-t-profitable-enough-for-big-pharma-to-make-more

MIT Laboratory for Financial Engineering. (2020). Estimates of clinical trial probabilities of success (PoS) - 2019Q4. Retrieved March 29, 2020, from https://projectalpha.mit.edu/pos/

Organisation for Economic Co-operation and Development. (2013). Health at a glance 2013: OECD indicators. https://doi.org/10.1787/health glance-2013-en

Pronker, E. S., Weenen, T. C., Commandeur, H., Claassen, E. H. J. H. M., \& Osterhaus, A. D. M. E. (2013). Risk in vaccine research and development quantified. PLoS ONE, 8(3), e57755. https://doi.org/10.1371/journal.pone.0057755

Rémy, V., Zöllner, Y., \& Heckmann, U. (2015). Vaccination: The cornerstone of an efficient healthcare system. Journal of Market Access \& Health Policy, 3(1), 27041. https://doi.org/10.3402/jmahp.v3.27041

Root, C. (2014, December 8). Biogen Idec moves aggressively, advances Alzheimer drug into phase 3. Clinical Leader. https://www.clinicalleader.com/doc/biogen-idec-moves-aggressivelyadvances-alzheimer-drug-into-phase-0001

Smietana, K., Siatkowski, M., \& Møller, M. (2016). Trends in clinical success rates. Nature Reviews Drug Discovery, 15(6), 379-380. https://doi.org/10.1038/nrd.2016.85 
Thomas, D. W., Burns, J., Audette, J., Carroll, A., Dow-Hygelund, C., \& Hay, M. (2016). Clinical development success rates 2006-2015. Biotechnology Innovation Organization (BIO). https://www.bio.org/sites/default/files/legacy/bioorg/docs/Clinical\%20Development\%20Success \%20Rates\%202006-2015\%20-\%20BIO,\%20Biomedtracker,\%20Amplion\%202016.pdf

Trefis. (2020). MRK revenues: How does Merck make money? Trefis. https://dashboards.trefis.com/no-login-required/qVh09Fjc/MRK-Revenues-How-Does-Merck-

Make-Money-

U.S. Department of Health and Human Services. (2017). Encouraging vaccine innovation: Promoting the development of vaccines that minimize the burden of infectious diseases in the 21st century. https://www.gpo.gov/fdsys/pkg/PLAW-114publ255/pdf/PLAW-114publ255.pdf

Vu, J., Kaplan, B., Chaudhuri, S., Mansoura, M., \& Lo, A. W. (2020). Financing vaccines for global health security. medRxiv. Cold Spring Harbor Laboratory Press. https://doi.org/10.1101/2020.03.20.20039966

Weir, J. P., \& Gruber, M. F. (2016). An overview of the regulation of influenza vaccines in the United States. Influenza and Other Respiratory Viruses, 10(5), 354-360. https://doi.org/10.1111/irv.12383

Wong, C. H., Siah, K. W., \& Lo, A. W. (2019a). Estimating clinical trial success rates and related parameters in oncology. SSRN Electronic Journal. https://ssrn.com/abstract=3355022

Wong, C. H., Siah, K. W., \& Lo, A. W. (2019b). Estimation of clinical trial success rates and related parameters. Biostatistics, 20(2), 273-286.

World Health Organization. (1980). The global eradication of smallpox: final report of the Global Commission for the Certification of Smallpox Eradication, Geneva, December 1979. https://apps.who.int/iris/handle/10665/39253

World Health Organization. (2020). Vaccines and diseases. https://www.who.int/immunization/diseases/en/

Young, R., Bekele, T., Gunn, A., Chapman, N., Chowdhary, V., Corrigan, K., Dahora, L., Martinez, S., Permar, S., Persson, J., Rodriguez, B., Schäferhoff, M., Schulman, K., Singh, T., Terry, R. F., \& Yamey, G. (2020). Developing new health technologies for neglected diseases: A pipeline portfolio review and cost model. Gates Open Research, 2, 23. https://doi.org/10.12688/gatesopenres.12817.3 


\section{Appendix}

\section{A1. An example of the path-by-path PoS calculations}

For clarity, we will walk our readers through some calculations using the example shown in Figure 6. In that figure, we see that 768 vaccine programs have conducted phase 1 testing whereas 1,178 vaccine programs have skipped phase 1 to go to phase 2 or 3 testing directly. This is not uncommon in vaccine development programs, where vaccine candidates move directly to the higher phases based on initial safety of the vaccine base (egg, etc.) after changing the virus within it. Among these 1,946 vaccine development programs, we know that 108 have yet to conclude phase 1 testing while 1,838 have completed phase 1 . Of these 1,838 programs, 1,517 have gone on to phase 2 while 321 have failed. In the notation introduced earlier, $\mathrm{N}_{1}=1,838$ and $\mathrm{N}_{2}=1,517$, yielding an estimate of $1,517 / 1,838$, or $82.5 \%$, for $\mathrm{PoS}_{12}$. Repeating the logic for the transitions between phase 2 and phase 3 , and between phase 3 and approval, gives $65.4 \%$ and $80.1 \%$ as estimates of $\mathrm{PoS}_{23}$ and $\mathrm{PoS}_{3 \mathrm{~A}}$ respectively.

In order to compute the probability of a vaccine development program making it all the way from phase 1 to approval, we consider only the vaccine development programs that have definite outcomes. In other words, we do not consider development programs that are 'in progress' in the denominator. In our example, the number of such programs is 1,178+768$108-178-108=1,552$. Since 615 programs made it to approval, the estimated $\operatorname{PoS}_{1 \mathrm{~A}}$ is $615 / 1552=39.6 \%$.

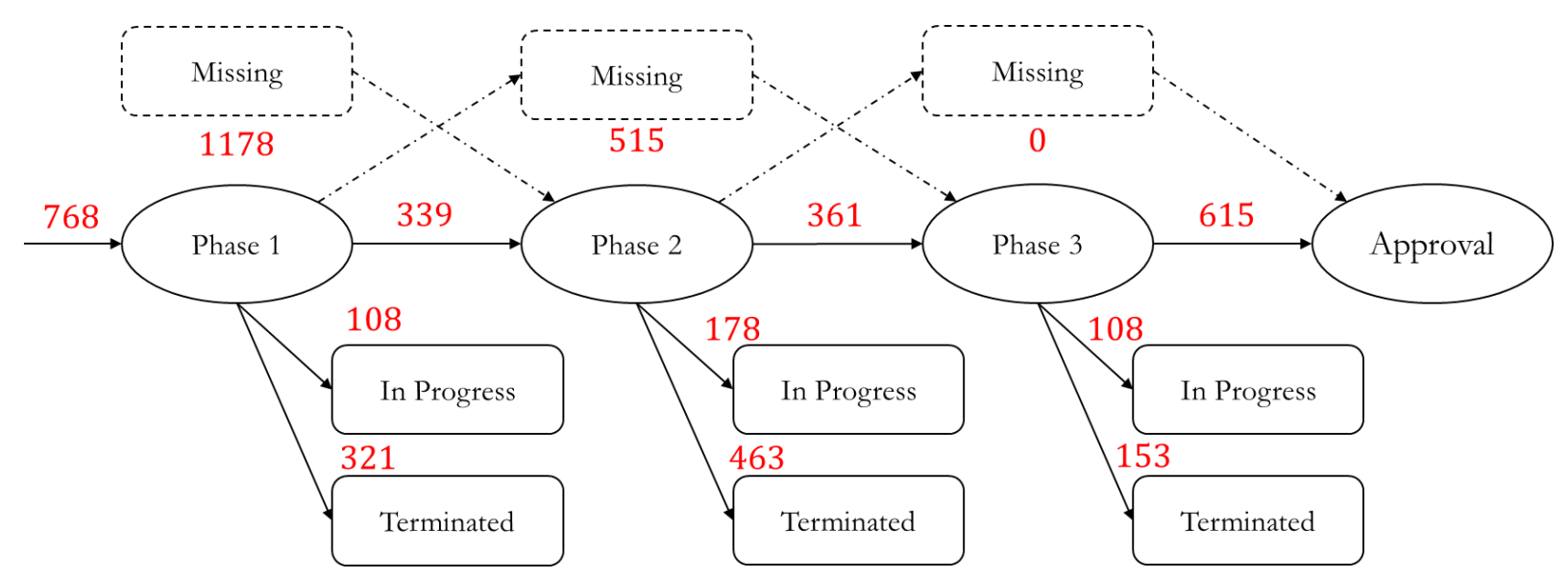

Figure 6. An example of the number of transitions computed, based on the data for industry-sponsored vaccine drug development programs. 


\section{A2. Differences between the path-by-path approach and the phase-by-phase approach}

The path-by-phase approach described in the main text carefully considers the drug development programs that are under active development and excludes them from the PoS calculations when necessary. As such, the overall probability of success, $\mathrm{PoS}_{1 \mathrm{~A}}$, is not the multiplication of $\mathrm{PoS}_{12}, \mathrm{PoS}_{23}$ and $\mathrm{PoS}_{3 \mathrm{~A}}$.

$$
\mathrm{PoS}_{1 A} \text { (path-by-path) } \neq \mathrm{PoS}_{12} \times \mathrm{PoS}_{23} \times \mathrm{PoS}_{3 \mathrm{~A}}
$$

In contrast, the phase-by-phase computation simply computes PoS $\mathrm{ij}_{\mathrm{ij}}$ using the equation:

$$
\mathrm{PoS}_{i j}=\prod_{x=i, \ldots, j-1} P o S_{x, x+1}
$$

In particular, the $\mathrm{PoS}_{1 \mathrm{~A}}$ is computed using the following formula:

$$
\left.\mathrm{PoS}_{1 A} \text { (phase-by-phase }\right)=\mathrm{PoS}_{12} \times \mathrm{PoS}_{23} \times \mathrm{PoS}_{3 \mathrm{~A}}
$$

The phase-by-phase approach is valid under some circumstances, such as when one does not have any development programs that are active in any of the phases in his database. This is easily seen if one simply set the number of 'in progress' development programs in all phases in Figure 6 to zero and recomputing the PoS.

The path-by-path approach can also obtain the same results as the phase-by-phase approach if one is willing to make an additional assumption: programs that are "in progress" in phase $i$ will transit to phase $i+1$ or to "terminated" with the same probability as going from phase $i$ to phase $i+1$, or from phase $i$ to "terminated", without "in progress" programs.

We will illustrate this with Figure 7, which shows the different states of a drug development program with hypothetical transitions from "in progress" states to the next phase or to the "terminated" state. Without considering "in progress" programs, the probability of transiting from phase 1 to phase 2 is $78.0 /(78.0+16.5)=82.5 \%$ and the probability of transiting from phase 1 into the "terminated" state is $17.5 \%$. Similarly, without considering "in progress" programs between phase 2 and phase 3, or phase 3 to Approval, the probabilities of transiting from phase 2 to phase 3, or phase 3 to approval are $65.4 \%$ and $80.0 \%$ respectively. If we set $a, b$ and $c$ to be $82.5 \%, 65.4 \%$ and $80.0 \%$ respectively, we will obtain a $\mathrm{PoS}_{1 \mathrm{~A}}$ of $43.2 \%$, which is exactly $\mathrm{PoS}_{12} \times \mathrm{PoS}_{23} \times \mathrm{PoS}_{3 \mathrm{~A}}$. In contrast, the path-bypath approach obtains a PoS $1 \mathrm{~A}$ of $39.6 \%$ as it does not make any assumptions and ignores programs that are "in progress" in either phase 1, phase 2 or phase 3.

We believe that our method of inferring unobserved clinical development stages and then applying the path-by-path approach is a better measure of the PoSs of clinical development programs as it does not underestimates the PoSs, and makes no assumption about the programs that are in active development. 


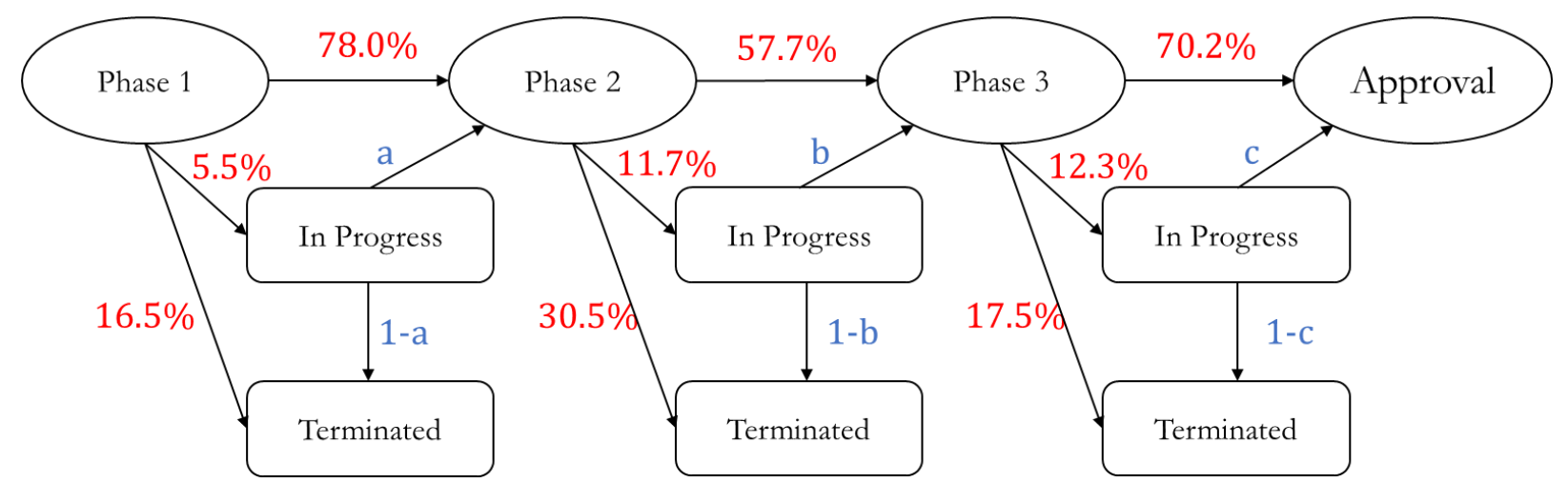

Figure 7. A Markov chain that includes hypothetical transitions from "in progress" states to the next phase or to the "terminated" state. 


\section{A3. List of transmission route and biological family for infectious diseases}

Table 9. List of transmission routes and biological family for the infectious diseases

\begin{tabular}{lll}
\hline Disease & Transmission Route & Biological Family \\
\hline Hepatitis B virus (HBV) & Human-human (Others) & Hepadnavirus \\
Other & Multiple or others & Multiple or others \\
Otitis Media & Multiple or others & Multiple or others \\
Bacterial Skin Infection & Multiple or others & Multiple or others \\
Sepsis & Multiple or others & Multiple or others \\
Human papillomavirus (HPV) & Human-human (Others) & Flaviviridae \\
Human immunodeficiency virus (HIV) & Human-human (Others) & Retroviridae \\
Intra-abdominal Infections & Multiple or others & Multiple or others \\
Onychomycosis & Multiple or others & Multiple or others \\
Clostridium difficile & Multiple or others & Clostridiaceae \\
Cytomegalovirus (CMV) Infection & Human-human (Others) & Herpesviridae \\
Hepatitis C virus (HCV) & Human-human (Others) & Flaviviridae \\
Respiratory Infections & Multiple or others & Multiple or others \\
Urinary Tract Infections & Multiple or others & Multiple or others \\
Rotavirus & Human-human (Others) & Reoviridae \\
Ebola & Human-human (Others) & Filoviridae \\
Marburg & Human-human (Others) & Filoviridae \\
Smallpox & Human-human (Airborne) & Poxvirus \\
Zika & Animal bites & Flaviviridae \\
Rabies & Animal bites & Rhabdoviridae \\
Yellow Fever & Animal bites & Flaviviridae \\
Chikungunya & Animal bites & Togaviridae \\
Norovirus & Contaminated food or water & Caliciviridae \\
Japanese Encephalitis & Animal bites & Flaviviridae \\
Non-tuberculous Mycobacteria (NTM) & Multiple or others & Multiple or others \\
West Nile Virus (WNV) & Animal bites & Flaviviridae \\
Middle East Respiratory Syndrome (MERS) & Human-human (Airborne) & Coronaviridae \\
Severe Acute Respiratory Syndrome (SARS) & Human-human (Airborne) & Coronaviridae \\
Monkeypox & Animal bites & Poxviridae \\
\hline & &
\end{tabular}




\section{A4. PoS Tables across all therapeutic areas}

We reproduce the probability of success across all therapeutic groups.

Table 10. The probabilities of success (PoSs) of industry-sponsored drug development programs across all therapeutic groups. The classification of vaccines used in this table is based on broader categories such as 'other viral vaccines' instead of the finer ones such as 'ebola' used in this paper, resulting in a slight difference in the computed PoSs. A: regulatory approval; P1: phase 1; P2: phase 2; P3: phase 3; SE = standard error.

\begin{tabular}{|c|c|c|c|c|c|c|c|c|c|c|c|c|c|}
\hline Disease & P1 Paths & $\mathrm{PoS}_{12}$ & S.E. & P2 Paths & $\mathrm{PoS}_{23}$ & S.E. & $\mathbf{P o S}_{2 \mathrm{~A}}$ & S.E. & P3 Paths & $\mathrm{PoS}_{3 \mathrm{~A}}$ & S.E. & $\mathbf{P o S}_{1 \mathrm{~A}}$ & S.E. \\
\hline Oncology & 27,600 & 65.5 & 0.3 & 10,650 & 37.7 & 0.5 & 6.9 & 0.3 & 2,597 & 28.2 & 0.9 & 3.9 & 0.1 \\
\hline Metabolic/Endoc & & 74.2 & 0.7 & 767 & 60.0 & 0.9 & 21.3 & 0.8 & 1,293 & 45.6 & 1.4 & 16.7 & 0.6 \\
\hline & & 74.3 & 0.8 & & 70.2 & 1.0 & 25.9 & 1.0 & & 48.8 & 1.4 & 21.4 & 0.8 \\
\hline CNS & 6,207 & 71.7 & 0.6 & 3,806 & 56.9 & 0.8 & 14.6 & 0.6 & 1,525 & 36.5 & 1.2 & 11.3 & 0.5 \\
\hline Autoimmune/Inflammation & 6,272 & 71.7 & & 3,704 & 52.7 & 0.8 & 17.3 & 0.7 & 1,332 & 48.0 & 1.4 & 13.1 & 0.5 \\
\hline Genitourinary & 1,103 & 71.4 & 1.4 & 737 & 61.6 & 1.8 & 25.0 & 1.7 & 352 & 52.3 & 2.7 & 19.3 & 1.3 \\
\hline Infectious Dise & 3,851 & 65.0 & 0.8 & 2,202 & 64.2 & 1.0 & 23.1 & 1.0 & 996 & 51.0 & 1.6 & 16.2 & 0.7 \\
\hline Ophthalmology & & 89.1 & 1.2 & 510 & 55.7 & 2.2 & 17.1 & 1.8 & 191 & 45.5 & 3.6 & 17.6 & 1.7 \\
\hline Vaccines (Infectious Disease) & 1,886 & 83.9 & 0.8 & 1,409 & 66.4 & 1.3 & 45.8 & 1.4 & 813 & 79.5 & 1.4 & 40.6 & 1.2 \\
\hline Total & 55,363 & 69.1 & 0.2 & 28,050 & 51.6 & 0.3 & 16.1 & 0.2 & 10,302 & 44.0 & 0.5 & 11.0 & 0.2 \\
\hline All except oncology & 27,763 & 72.7 & 0.3 & 17,400 & 60.1 & 0.4 & 21.8 & 0.3 & 7,705 & 49.3 & 0.6 & 17.1 & 0.3 \\
\hline
\end{tabular}

